\title{
Fiber Optic Plasmonic Sensors: Past, Present and Future
}

\author{
Sachin K. Srivastava ${ }^{1, *}$ and Banshi D. Gupta ${ }^{2}$ \\ ${ }^{I}$ Department of Electro-Optic Engineering, Ilse Katz Institute for Nanoscale Science and Technology, Ben-Gurion Uni- \\ versity of the Negev, Be'er Sheva, Israel \\ ${ }^{2}$ Physics Department, Indian Institute of Technology Delhi, New Delhi-110016, India
}

\begin{abstract}
We review various fiber optic sensors utilizing both the propagating and localized surface plasmon resonance techniques. The utilization of optical fibers in plasmon based sensing has provided several advantages in sensing of various physical, chemical and biochemical parameters. The article starts with a brief introduction of the propagating surface plasmon resonance (SPR) and localized surface plasmon resonance (LSPR), presents the review of some of the past studies on SPR and LSPR based state-of-the-art fiber optic sensors and finally ends with the future scope of the plasmonics based fiber optic sensors. In addition, we discuss some latest results on the plasmon enhanced whispering gallery mode sensors, which require tapered optical fibers for excitation. The present review may provide the researchers a rigorous and organized literature for the understanding of the basics, utility and trends of fiber optic plasmonic sensor in a chronological order.
\end{abstract}

Keywords: Fiber optics, sensors, surface plasmons, localized surface plasmons.

\section{INTRODUCTION}

Plasmonics has been fascinating researchers for last three decades due to its overwhelming and structure specific optical properties. Both, the physics of surface plasmons as well as their applications in various fields have got wide attention due to interesting optical phenomena involved. The field of plasmonics, primarily applied to the sensing and waveguiding applications, has now broadened to SPACERs, treatment of cancers, nanoantennas, and nanofocussing, etc. [1-4]. The possibility of super resolution, far beyond the diffraction limit, which plasmonic structures posses, makes them extremely useful in ultrahigh resolution and detection of single biomolecular interactions $[5,6]$. The state of the art fabrication and characterization techniques have played crucial role in advent of novel plasmonic and nanoplasmonic structures, which posses multi-resonant characteristics [7]. Collaboration of plasmonics with optical fibers has resulted in the miniaturized sensors with low cost, high performance, light weight and portability. The additional advantage due to optical fibers is the capability of remote sensing and online monitoring, which make the sensor useful even in hazardous environments.

The organization of the present review is as follows: We have subdivided the article into two broad areas; first, the propagating surface plasmon resonance based fiber optic sensors and the second, localized surface plasmon resonance based sensors. Throughout the article, we have used the term 'surface plasmon resonance (SPR)' for propagating surface plasmon resonance. The review starts with a brief history of surface plasmons (Section 2.1) and then presents a general

\footnotetext{
*Address correspondence to this author at the Department of Electro-Optic Engineering, Ilse Katz Institute for Nanoscale Science and Technology, Ben-Gurion University of the Negev, Be'er Sheva, Israel,

Tel: 972-8-64-285-97, Fax: +972-(0)8-6479494;

E-mail: sachinchitransh@gmail.com
}

overview of its physics and sensing applications (Section 2.2). In section 2.3 , we discuss some of our studies on fiber optic SPR sensors, followed by influence of certain parameters on their performance (Section 2.4) and methods to enhance their sensitivity (Section 2.5). Section 3 is devoted to localized surface plasmon resonance based sensors, in which, we again start with a brief history, general overview and then fiber optic LSPR sensors (Sections 3.1, 3.2, and 3.3). In section 3.4, the influence of temperature over the performance of a fiber optic LSPR sensor has been presented. Section 3.5 discusses the enhancement of sensitivity. In section 4 , we have discussed some of the recent literature to point towards the new directions of future research. We have then added two appendices to present the mathematical formulation of surface plasmons, and localized surface plasmons to calculate the total power at the output end of optical fiber.

\section{SURFACE PLASMONS}

\subsection{Brief History}

The surface plasmons have a history going back more than about a hundred years. The first observation of surface plasmons was made by Wood in 1902 [8]. It was observed that when polarized light is incident on a mirror with the diffraction grating on its surface, an anomalous pattern of dark and bright bands appears in the reflected light. However in 1907, Zenneck theoretically formulated a special surface wave solution of the Maxwell's equations and predicted that radio frequency surface electromagnetic waves occur at the interface of a lossy dielectric or a metal and a lossless dielectric [9]. He further suggested that the lossy part of the dielectric constant of the metal was responsible for the electromagnetic surface wave at the interface. In 1909, the field amplitudes of the surface waves postulated by Zenneck, were found to vary inversely as the square root of the distance from the dipole [10]. In 1941, Fano concluded that the anomalies reported by Wood (1902) were because of the 


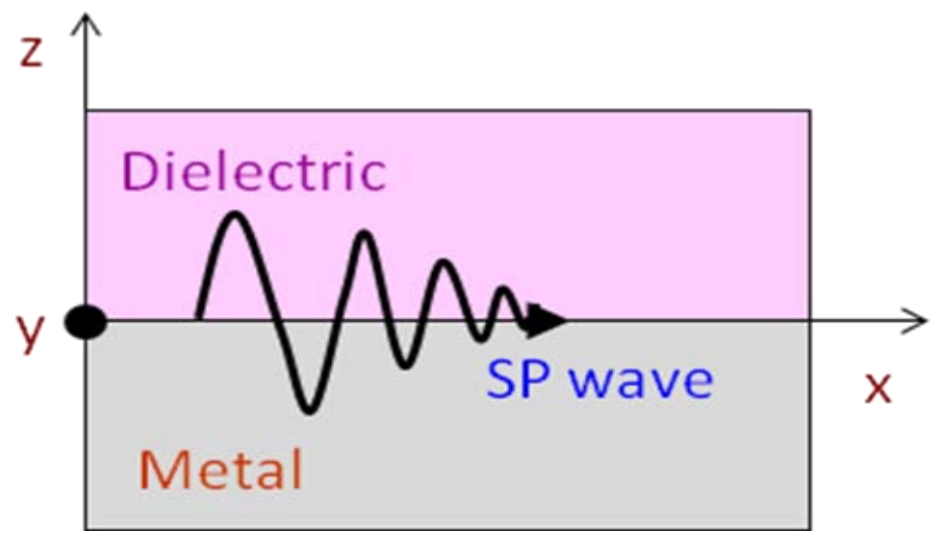

Fig. (1). Schematic of a surface plasma wave at a metal-dielectric interface.

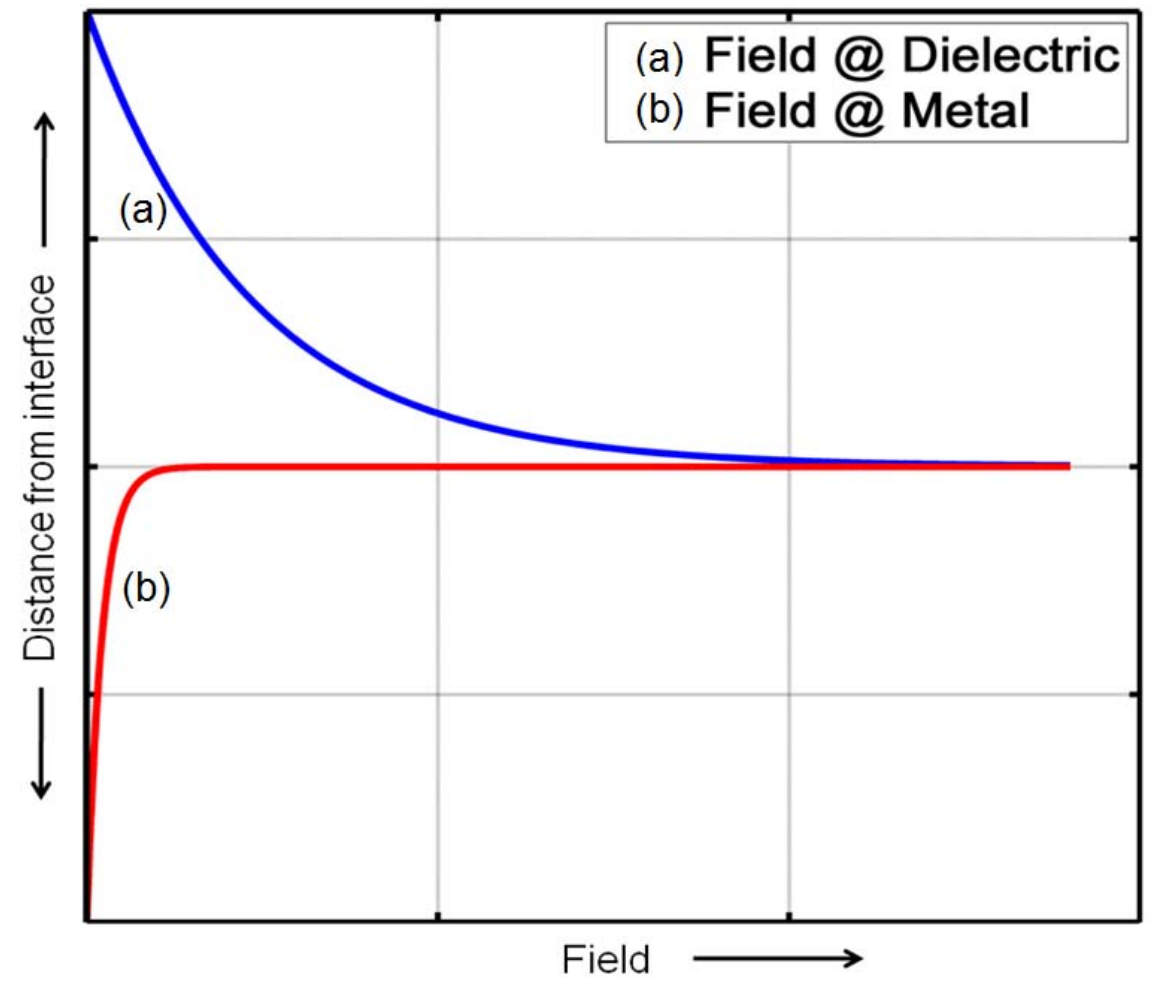

Fig. (2). Variation of the field of SP wave across the metal-dielectric interface.

excitation of surface waves on the surface of the diffraction grating [11]. Ritchie, in 1957, also predicted the excitation of the surface plasmons on the metal surface [12]. In 1959, Turbadar observed a large drop in the reflectivity when illuminating thin metal films on a substrate, but did not link this effect to the surface plasmons [13]. In 1960, Powell and Swan observed the excitation of surface plasmons at the metal surface using electrons [14]. Soon after, in 1960 only, it was concluded by Stern and Ferrell that the electromagnetic waves at the metallic surface possessed electromagnetic radiation intermingled with surface plasmons [15]. In 1968, Otto explained Turbadar's results and demonstrated that the drop in the reflectivity in attenuated total internal reflection is due to the excitation of the surface plasmons [16]. However the Otto configuration was not very much suitable for the practical point of view due to the finite gap between the metal layer and the prism base. In 1971, Kretschmann modified the Otto configuration by removing the space between the metal layer and the prism [17]. This scheme has been found to be better than previous configurations, from application point of view. Since then the Kretschmann configuration has been utilized in various sensing applications.

\subsection{General Overview}

Surface plasmons are the quanta of charge density oscillations at a metal dielectric interface. These oscillations get excited when the energy and wave-vector of incident light at the interface becomes equal to that of surface plasmon modes supported by the interface. As a result of these oscillations, under the action of the rapidly changing electromagnetic field, a wave, called surface plasma wave, gets generated, which travels along the interface and its field amplitude decays exponentially in both the metal and the dielectric medium (Fig. (1) and Fig. (2)). It can be observed in Fig. (2) that the field amplitude decay in metal (curve (b)) is faster than that in the dielectric medium (curve (a)). This can be 


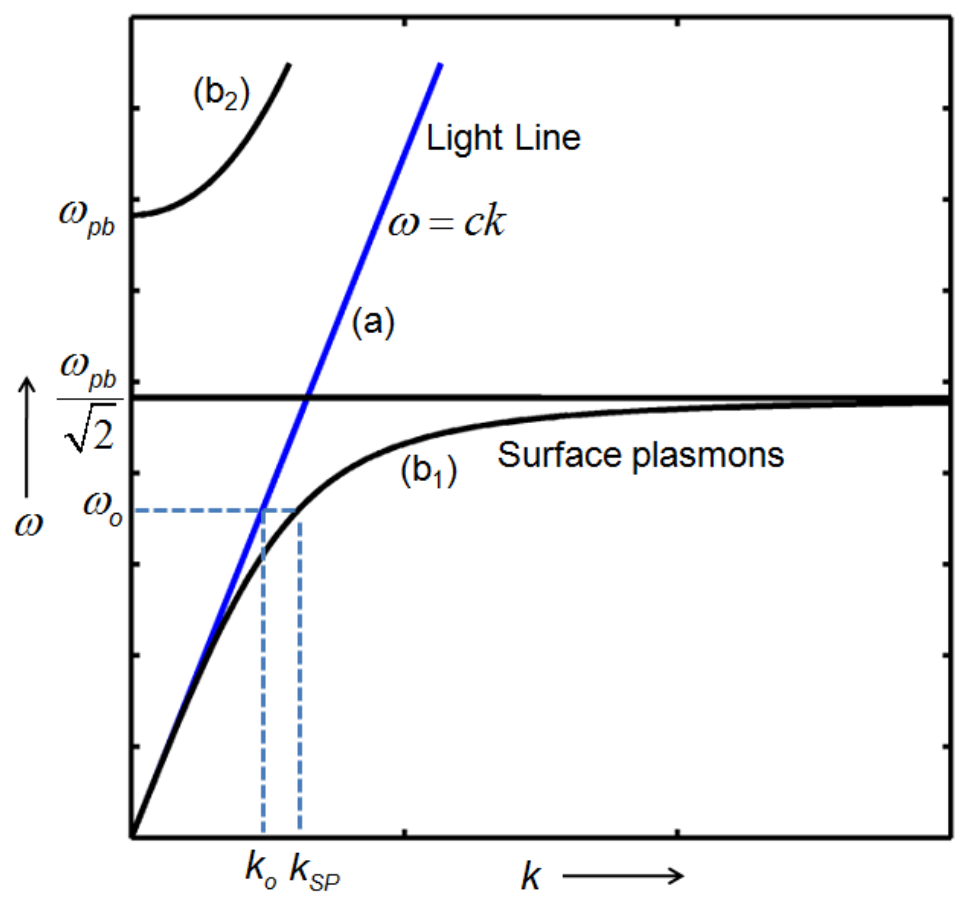

Fig. (3). Dispersion curves for the surface plasmon wave and the direct light.

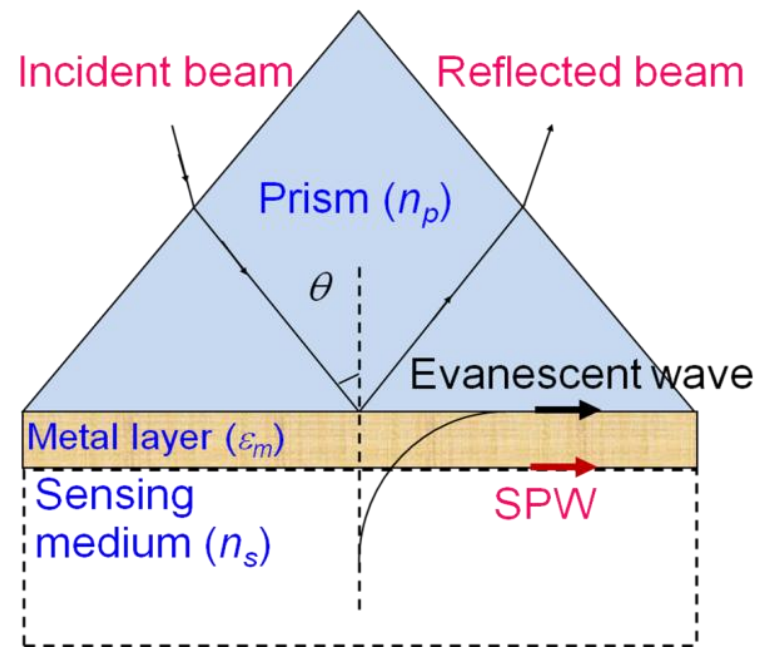

Fig. (4). A Schematic of Kretschmann Configuration.

attributed to the imaginary part of the metal dielectric function. Also, these waves are transverse magnetically (TM) polarized and get excited by only TM polarized light.

In general, surface plasmons cannot be excited at a metal-dielectric medium interface by directly shining it by a beam of light, because the wave-vector of the light in the dielectric medium is always smaller than that of the surface plasmons at the interface. It can be better understood by plotting the dispersion curves for a light wave and a surface plasmon wave. The dispersion curves for both the surface plasmons at air-gold interface and light in air have been plotted in Fig. (3). The straight line (a) in Fig. (3) corresponds to light line following the dispersion relation $\omega=c k$, the curve $\left(b_{1}\right)$ corresponds to surface plasmons bound to the interface, while $\left(b_{2}\right)$ corresponds to radiative plasmons. It can be observed that for a light wave of frequency $\omega_{o}$, the wave vector of surface plasmon is greater than that of light. Therefore light directly incident from the dielectric medium at the metal dielectric interface cannot excite surface plasmons [18]. For frequencies greater than the plasma frequency $\omega_{p b}$ (region $\left(b_{2}\right)$ ), the plasmonic modes are called radiative and the metal becomes transparent.

There are special techniques for the excitation of surface plasmons at a metal dielectric interface, in which the wavevector of the incident light is increased to match with surface plasmons. Two most general techniques are named as Otto and Kretschmann configurations. Some periodically structured surfaces have also been used for increasing the wavevector of the incident light. In such a case, however, the dielectric medium needs to be quite transparent, so that light can be collected in reflection mode. Fig. (4) shows the schematic of a typical Kretschmann configuration. In a Kretschmann configuration, the base of a high refractive index prism is coated with a very thin layer of nobel metal, 


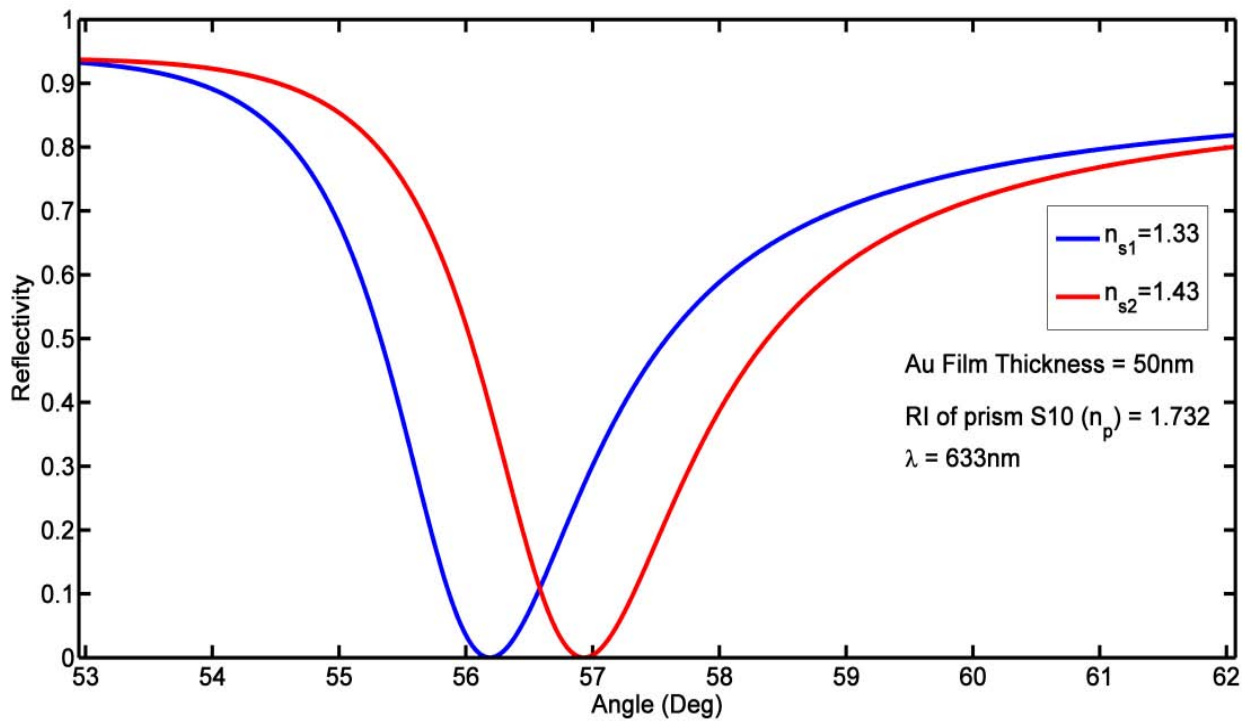

Fig. (5). SPR curves for a Kretschmann configuration, with prism made of S10 glass, gold film of $50 \mathrm{~nm}$ thickness.

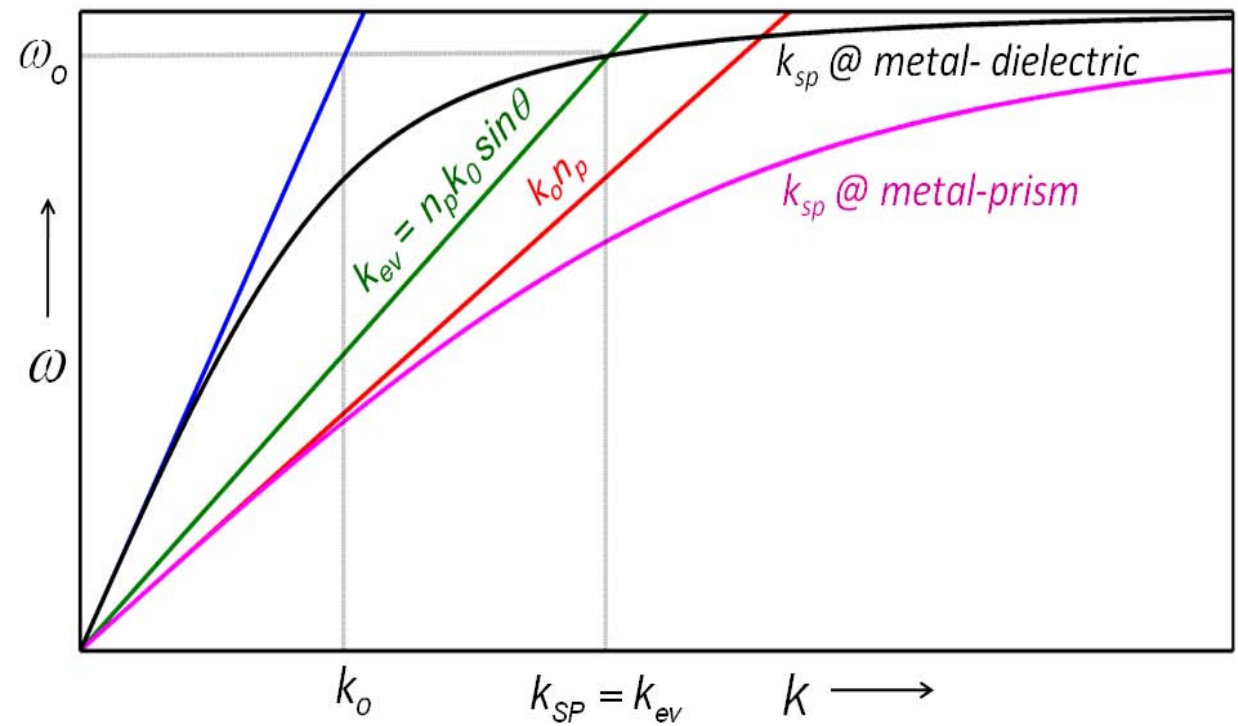

Fig. (6). SPR dispersion curves for a Kretschmann like configuration.

which is further surrounded by the dielectric medium. The light reflected off the other face is fed to a detector. The evanescent field at the prism metal interface excites the surface plasmons at the metal-dielectric interface, when the frequency and wave-vector of the incident light becomes equal to that of surface plasmon mode. This is termed as surfce plasmon resonance (SPR).

As a result of the resonance, a dip is obtained in the reflected power, corresponding to a particular angle at which the wave vectors match. Fig. (5) shows the variation of reflectivity with angle for dielectric media of two different refractive indices. It is observed that the resonace position changes with a change in the refractive index of the dielectric medium. With an increase in the refractive index of the surrounding medium, the resonance angle increases. This property of surface plasmons is utilized in sensing applications.

Fig. (6) shows the dispersion curves of surface plasmons and incident light for a Kretschmann like configuration. It is observed that for a certain angle of incidence at the prismmetal interface, for a monochromatic light, the wave-vectors of both the surface plasmons at the metal-dielectric interface and incident light match. However, light incident through the prism cannot excite surface plasmons at the metal-prism interface, as the wave vector of the surface plasmon mode at this interface is greater than that of light in the prism. Mathematically, it can be understood as follows: The wave vector of surface plasmon $\left(k_{s p}\right)$ is given by the following relation

$k_{s p}=k_{o}\left(\frac{\varepsilon_{m} \varepsilon_{s}}{\varepsilon_{m}+\varepsilon_{s}}\right)^{1 / 2}$

where, $k_{o}$ is the wave vector of light in free space and $\varepsilon_{m}$ and $\varepsilon_{s}$ are the dielectric constants of the metal and the dielectric medium, respectively. The wave vector of the evanescent wave is given by following relation

$k_{e v}=k_{p} \sin \theta=\frac{\omega}{c} \sqrt{\varepsilon_{p}} \sin \theta$ 


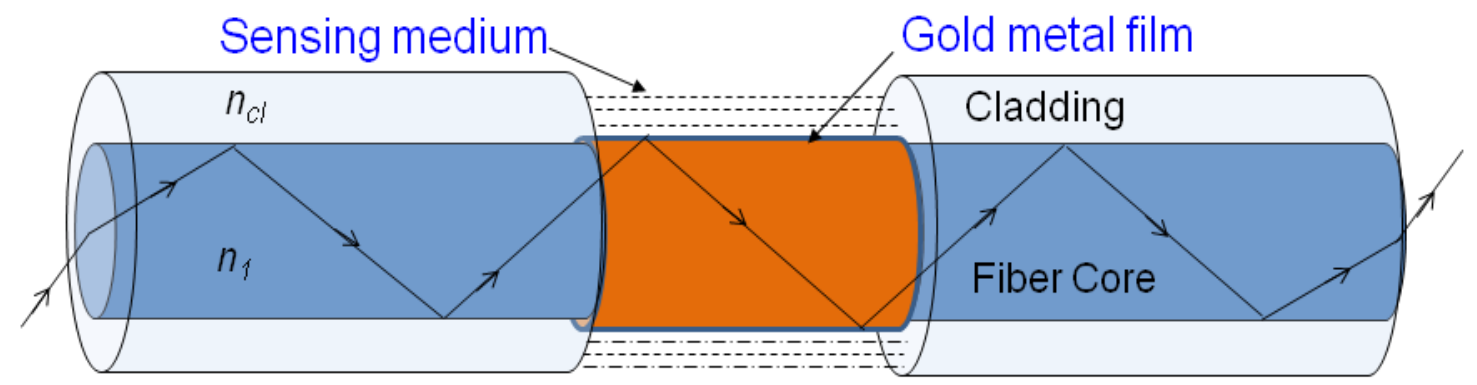

Fig. (7). Schematic of a fiber optic SPR sensor.

where $k_{p}$ is the wave vector of the incident light in prism with dielectric constant $\varepsilon_{p}, \omega$ is the angular frequency and $c$ is the speed of light in free space. The complete transfer of energy takes place when the wave vector of evanescent wave and the surface plasmon wave become equal, or the following relation is satisfied

$$
\frac{\omega}{c} \sqrt{\varepsilon_{p}} \sin \theta_{r e s}=\frac{\omega}{c}\left(\frac{\varepsilon_{m} \varepsilon_{s}}{\varepsilon_{m}+\varepsilon_{s}}\right)^{1 / 2}
$$

\subsection{Fiber Optic SPR Sensors}

Among the first few reports on fiber optic SPR sensors, the work reported by Villuendas and Palayo got attention of researchers [19]. Their work consisted of experimental results on aqueous sucrose solutions for sensitivity and dynamic range evaluation. Soon after, a four layer fiber optic SPR sensor was reported with enhanced sensitivity and dynamic range [20]. Around the same time, a single mode optical fiber SPR sensor with SPR excitation on the tip was reported [21]. Among these reports, the one reported by Jorgenson and Yee got the most attention, due to a complete theoretical and experimental study of fiber optic SPR sensors, with an application in chemical sensing [22]. Jorgenson and Yee replaced the prism of the Kretschmann configuration by the core of an optical fiber and excited surface plasmons by the evanescent field at the core-cladding interface. Their sensor was well accepted because SPR sensors utilizing optical fibers have been found to provide multiple advantages over prism based sensors. These sensors are simple in design, miniaturized, low cost, highly sensitive and accurate. Use of optical fiber further adds the advantage of online monitoring, remote sensing and freedom from electric shocks and electromagnetic interferences. The compatibility of gold with various biological species and functional groups makes these sensors useful for biosensing applications. Since then, various fiber optic sensors have been reported [23]. A number of attempts have been made to enhance the sensitivity of these sensors by various probe designs and additional layers $[24,25]$. These sensors have been employed in sensing of different chemicals and biochemicals and environmental and health monitoring [26-28]. Bimetallic and alloy films have also been used to enhance the performance [29, 30]. Some theoretical reports have presented the collaboration of plasmonic structures with fiber Bragg gratings (FBGs) and long period gratings (LPGs) [31]. However, the SPR based FBG sensors have not yet been realized probably due to experimental difficulties and still optimization of certain parameters is required before fabrication. The fiber optic interferometric sensors utilizing SPR have also been studied in Fabry-Perot configuration [32].

In a fiber optic SPR sensor, generally, the cladding of a small portion of the optical fiber is removed from the middle and coated with a thin layer of metal. The dielectric medium to be sensed further surrounds the metal coated region as shown in Fig. (7). Polychromatic light launched from one end of the optical fiber gets guided into it by the phenomenon of total internal reflection at the core-cladding interface. The evanescent field of the light guided into the optical fiber excites surface plasmons at the metal coated region. As a result of SPR, the power transmitted off the output end of the fiber shows a dip in the transmission spectrum. This happens due to the absorbance of the light of a particular wavelength by surface plasmons. When the refractive index of the dielectric medium around the sensing region is changed, the position of the dip in the transmission spectrum gets changed.

A schematic of an experimental setup to characterize a fiber optic SPR sensor is shown in Fig. (8). Before launching of light in the optical fiber, the sensor probe is fixed in a glass flow cell having inlet and outlet facilities for liquid sensing samples. Next we present some of the studies on fiber optic SPR sensors.

\section{(a) Detection of Low Water Contents in Ethanol}

Ethanol is seen as a prospective fuel which may replace the dependence on conventional fossil fuels. It has been given more importance due to limited amount of fossil fuels and pollution concerns. However, ethanol must be at least 93\% pure to be used as a fuel [34]. Apart from that, ample amounts of ethanol are required in medical industry, biological sciences and organic chemistry, where it is needed in purest forms. The ethanol is mostly manufactured by its extraction from molasses and contains water as a miscible impurity. Hence, it is very important to detect the amount of water in ethanol and remove it. Water in ethanol mixtures of varying water percentages in ethanol were prepared and corresponding SPR spectra were recorded by using the experimental setup shown in Fig. (8). Fig. (9) shows the recorded SPR spectra for different percentages of water in ethanol [33]. It is observed that with the increasing water percentage in ethanol, the SPR spectra first show a red shift and then after a certain water percentage, they shift toward blue wavelength region. This kind of behavior is totally confusing as the refractive index variation must vary uniformly with the increase of water percentage. The reason behind such a behavior is attributed to the fact that at small water percentages 


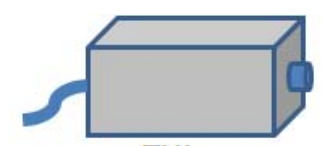

THL<smiles>COCCCCCCO</smiles><smiles></smiles>

3D-TS
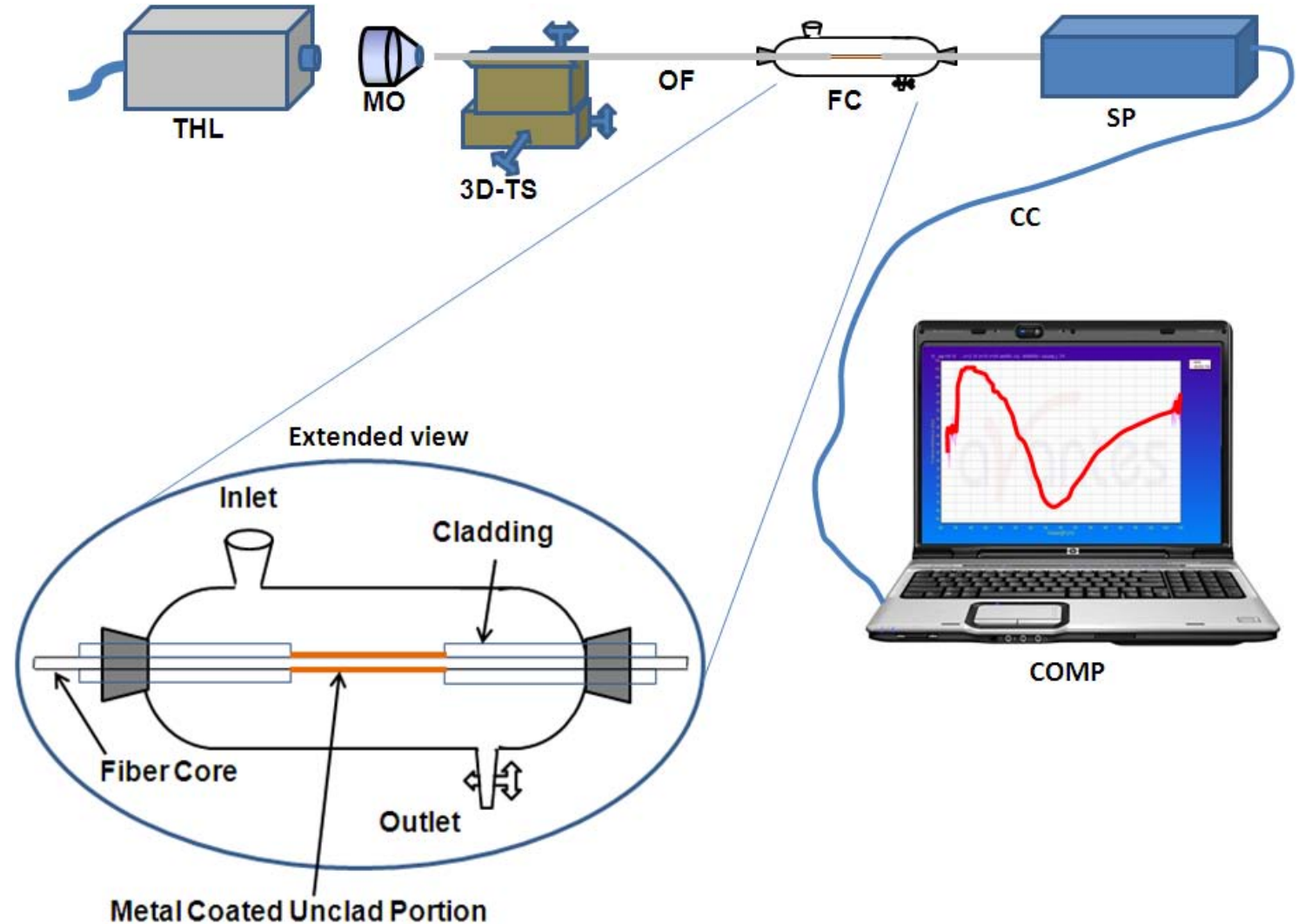

CC

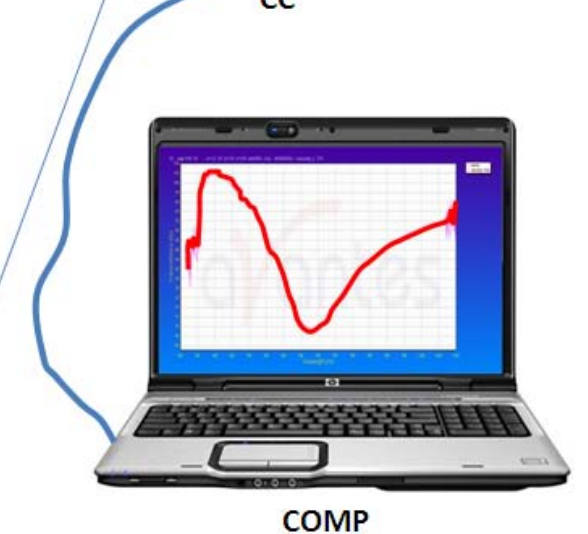

COMP

Fig. (8). Schematic of the experimental setup used for the study of SPR spectra. THL: tungsten-halogen lamp, MO: microscope objective, 3D-TS: three dimensional translation stage, OF: optical fiber, FC: flow cell, SP: spectrometer, CC: connecting cable, COMP: computer. (Reprinted with permission from Elsevier [33]).

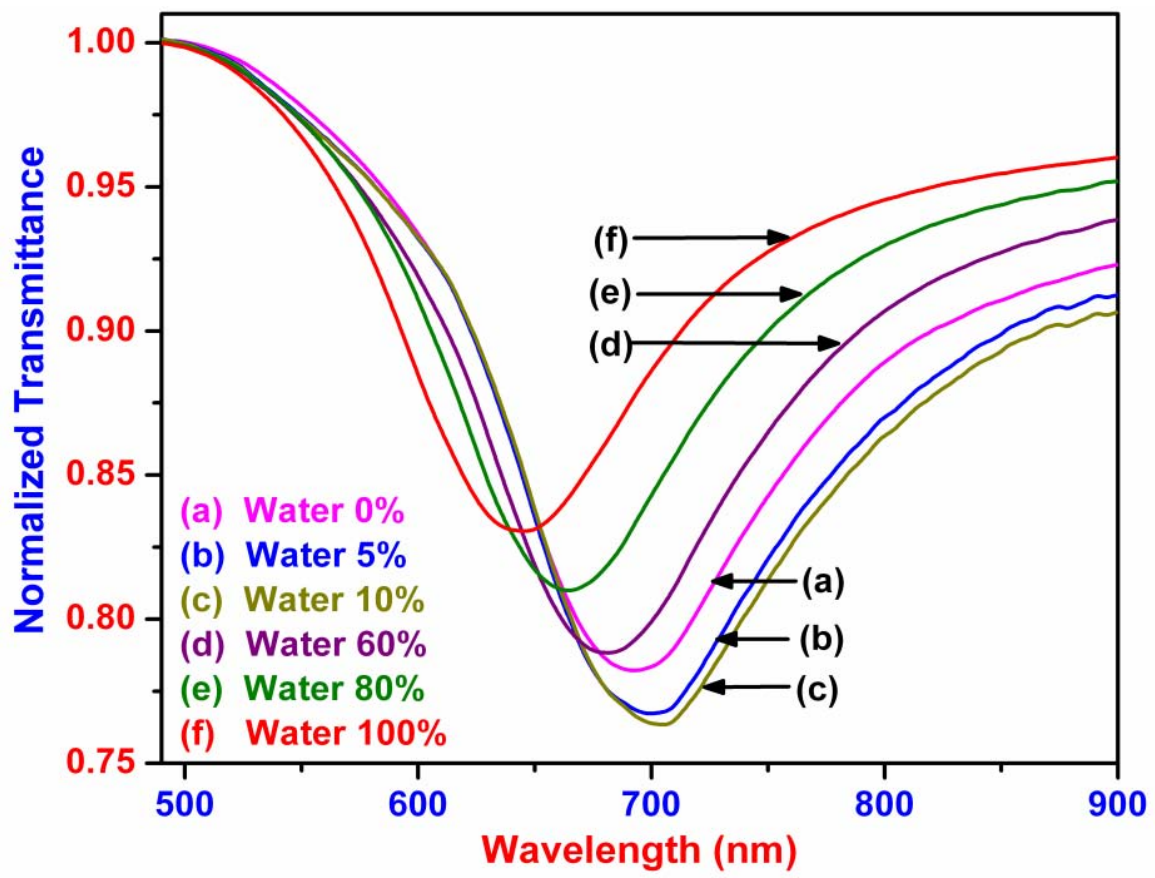

Fig. (9). SPR spectra for different water contents in ethanol-water mixture (Reprinted with permission from Elsevier [33]). 


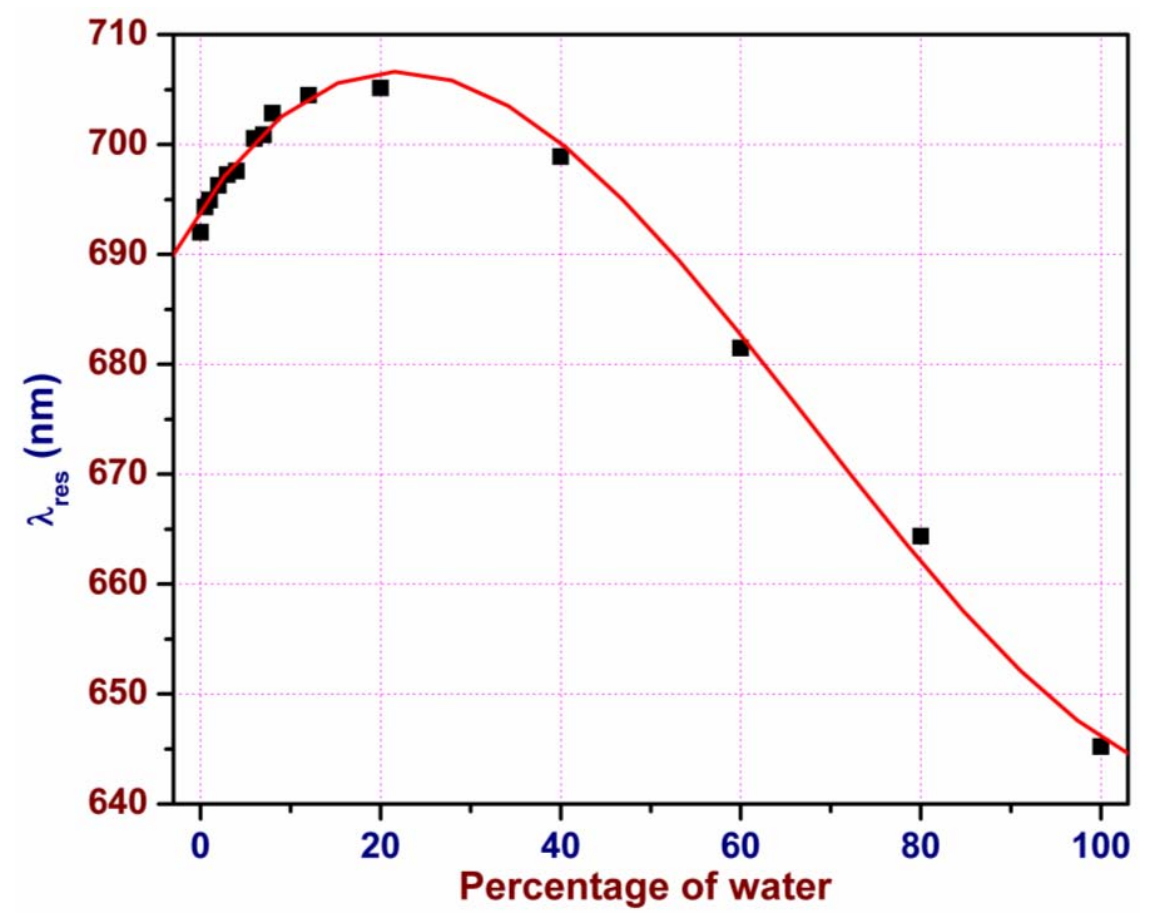

Fig. (10). Variation of resonance wavelength with water content in ethanol-water mixture (Reprinted with permission from Elsevier [33]).

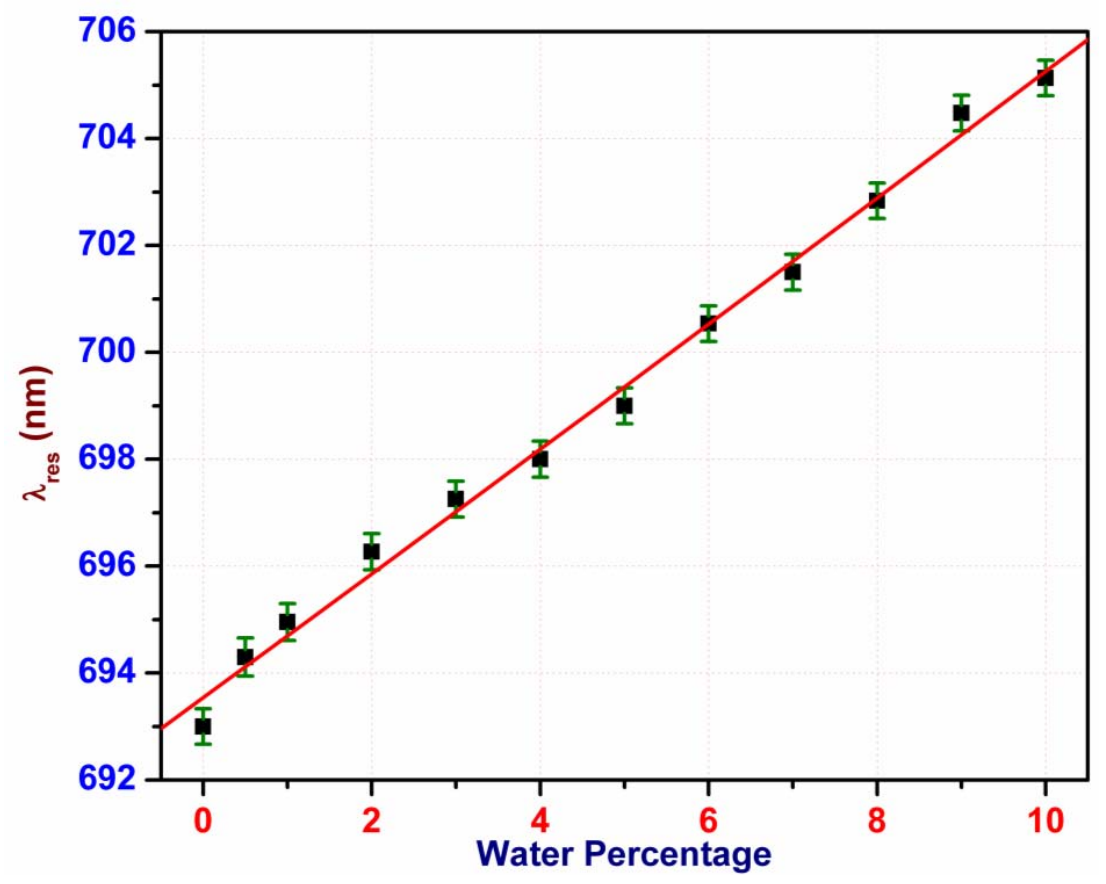

Fig. (11). Variation of resonance wavelength with water content in ethanol-water mixture for the range 0 to $10 \%$ (Reprinted with permission from Elsevier [33]).

in ethanol, small clusters of ethanol-water are formed due to local hydrogen bonding [35]. As the water percentage increases, the density of these clusters increases and hence the refractive index rises a bit. After a certain water percentage, further addition of water leads to the uniform hydrogen binding throughout the mixture, which results in disappearance of the clusters, giving rise to decrease in the refractive index. The shift in resonance wavelength with water percentage is plotted in Fig. (10) [33].
It can be seen that either the rising phase or the falling phase of the characteristic curve can be used for sensing, as for a certain range, the same resonance wavelength corresponds to two water percentages. Since, ethanol is needed in purest forms for its various applications; the region of interest is the rising phase of the characteristic curve. The variation of resonance wavelength with water percentage up to $10 \%$ water in ethanol is shown in Fig. (11) [33]. It is observed that the response of the sensor in this range of interest 


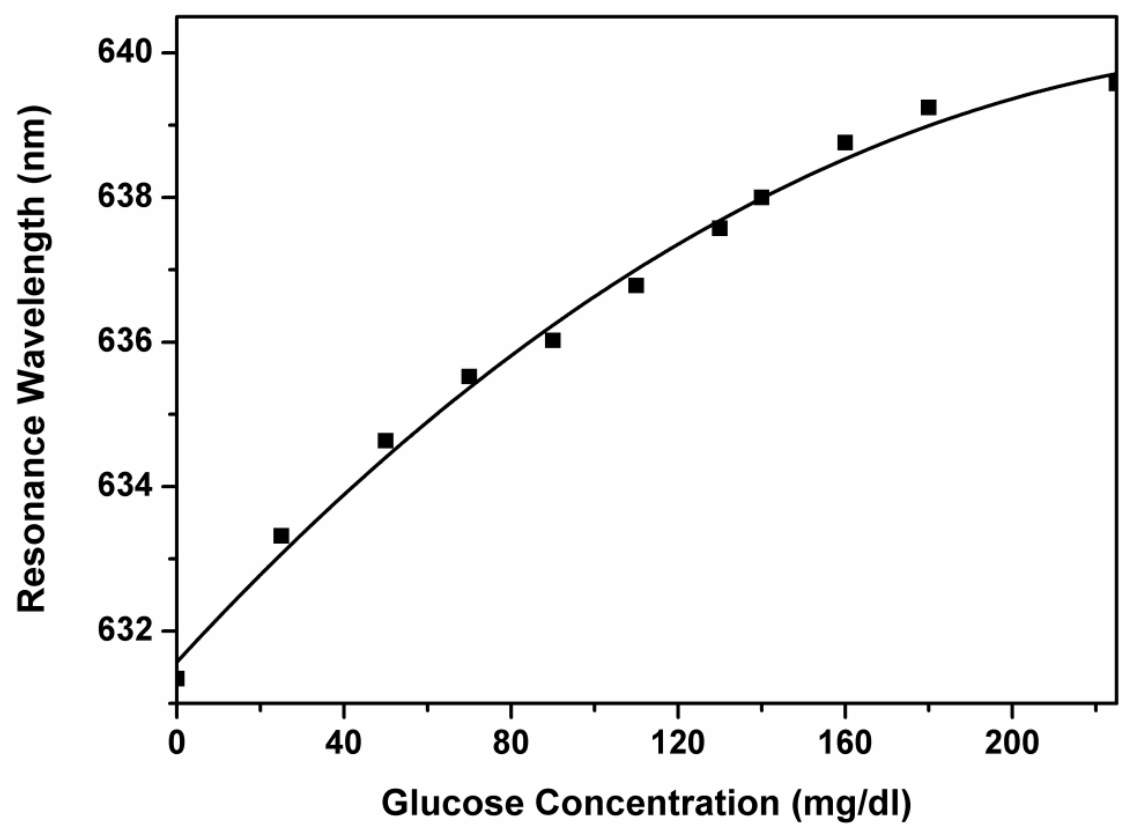

Fig. (12). Variation of resonance wavelength with glucose concentration (Reprinted with permission from SPIE [36])
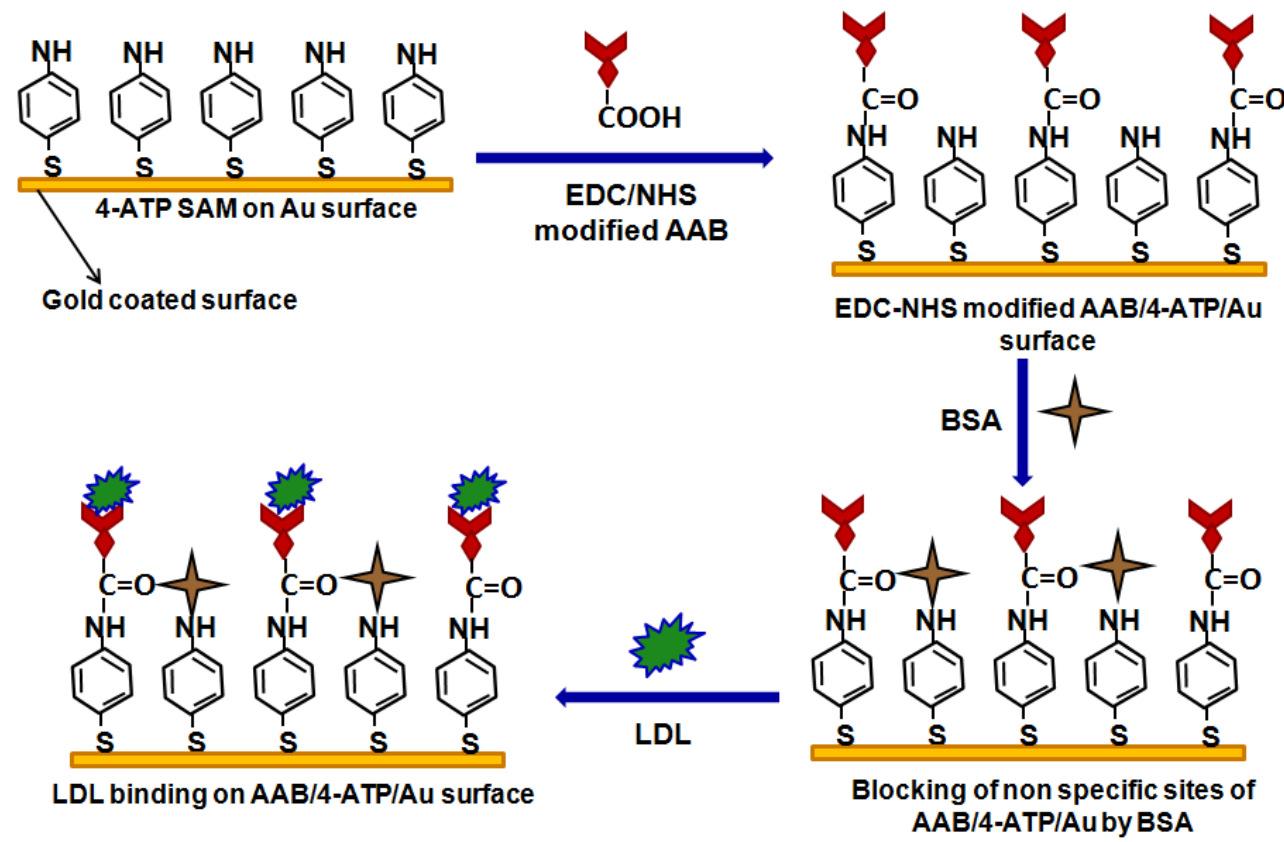

Fig. (13). Schematic of stepwise preparation of AAB/4-ATP/Au modified fiber surface for binding of LDL. (Reprinted with permission from IEEE [37]).

is linear. The sensitivity calculated by the slope of the plot was $1.149 \mathrm{~nm} /$ water \% in ethanol [33].

\section{(b) Glucose and Low Density Lipoprotein Biosensors}

Fiber optic SPR sensors have rigorously been used for biosensing applications [26-28]. To fabricate a biosensor, receptors of desired molecule are generally immobilized over the metal film and suitable analytes are then flown over the immobilized portion. A glucose sensor was fabricated by immobilizing a self assembled monolayer (SAM) of glucose oxidase over the gold layer [36]. Fig. (12) shows the variation of SPR wavelength with glucose concentration. It is observed that the SPR wavelength increases with the increase in the glucose concentration. The sensitivity of the sensor was found to be $0.0366 \mathrm{~nm} /(\mathrm{mg} / \mathrm{dl})$. Similar efforts were made to fabricate a SPR based fiber optic low density lipoprotein (LDL) sensor [37]. The detection of LDL is a crucial issue, as it is bad cholesterol and is responsible for the heart diseases. The sensor was fabricated by immobilizing anti-apolipoprotein $\mathrm{B}$ (AAB) over gold. Fig. (13) shows the stepwise fabrication of the sensor by SAM formation over gold [37]. The gold coated optical fiber was first functionalized by 4- Aminothiophenol (4-ATP), which was further immobilized by antiapolipoprotein $\mathrm{B}(\mathrm{AAB})$. Before the 


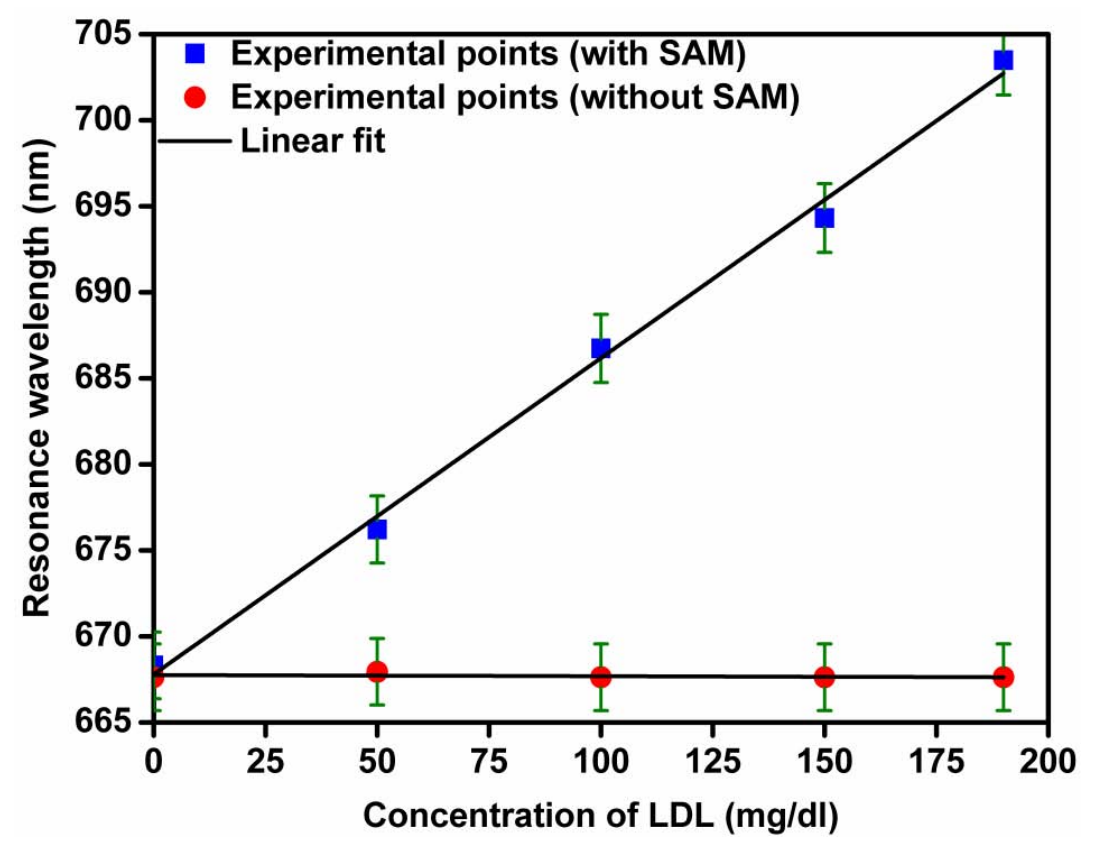

Fig. (14). Variation of resonance wavelength with concentration of LDL for fiber optic SPR probes with and without SAM layers. (Reprinted with permission from IEEE [37]).

immobilization of $\mathrm{AAB}$ over 4-ATP/Au surface, AAB was first cross-linked by EDC (N-ethyl-N-(3-dimethylaminopropyl carbodimide)-NHS (N-hydroxysuccinimide) chemistry to increase the coupling efficiency by $10-20$ folds [38]. The conjugation of AAB with EDC-NHS allowed direct immobilization of $\mathrm{AAB}$ on 4-ATP/Au surface. The prepared $\mathrm{AAB} / 4-\mathrm{ATP} / \mathrm{Au}$ optical fiber probe was then incubated in BSA $(2 \mathrm{mg} / \mathrm{ml})$ solution for one hour at room temperature to block those 4-ATP sites which remain unbound by AAB and are unspecific for the binding of LDL. This step helps in remarkably reducing the binding of any foreign element which may give false response and makes the sensor specific to low density lipoprotein (LDL) only. The response of the sensor with and without the SAM layers is shown in Fig. (14) [37]. It is observed, that the response of the sensor is linear in the range of interest. The experimental curves for the sensor without SAM layer were obtained by a 'control experiment' performed to check the role of SAM layer in the sensing. It was observed that there is no change in resonance wavelength with change in LDL concentration for a sensor without SAM layer. The response of the sensor in the range of operation was found to be linear. The sensitivity of the sensor, calculated from the response curve, was found to be $0.18387 \mathrm{~nm}$ per $\mathrm{mg} / \mathrm{dl}$ concentration of LDL.

\subsection{Influence of Parameters other than Refractive Index}

From the above, it is clear that a change in refractive index of the sensing medium leads to a change in the surface plasmon resonance wavelength. This gives rise to a plain curiosity that is it only the refractive index of the dielectric medium which changes the plasmon resonance or some other parameters can affect the plasmon resonance condition. Lioubimov et al studied the effect of varying electric potential on the performance of a SPR sensor [39]. The effect of temperature on the performance of a fiber optic SPR sensor was studied and the effects of temperature on sensitivity and signal to noise ratio (SNR) for two plasmonic metals silver and gold were discussed [40]. The influence of various dopants in the optical fiber core on the performance of fiber optic SPR sensor was studied [41]. It was concluded that the sensitivity of the sensor increases with the increase in doping concentration of $\mathrm{B}_{2} \mathrm{O}_{3}$, while the SNR remains unaffected. Further, the influence of skew rays on the performance of a fiber optic SPR sensor was studied and it was concluded that skew rays degrade the performance [42]. The influence of ions on the performance of a fiber optic SPR sensor has also been studied [43]. Fig. (15) shows the variation of surface plasmon resonance wavelength with refractive index for four ionic and two non-ionic aqueous solutions. It was found that, for the same refractive index of the sensing medium, the shift in resonance wavelength is greater for ionic media than that for non-ionic ones. Also, the shift in resonance wavelength was found to be different for different ionic media. Thus, the ions are found to affect the SP resonance condition. To confirm the role of the ions, the variation of ion concentration with refractive index was studied. It was found that, for the same value of the ion concentration, the refractive index of $\mathrm{Na}_{2} \mathrm{SO}_{4}$ and $\mathrm{MgCl}_{2}$ is greater than $\mathrm{NaCl}$ and $\mathrm{KCl}$ as shown in Fig. (16) [43]. Thus, it was concluded that the shift in resonance wavelength increases with an increase in the ion concentration in the sample solution.

\subsection{Enhancement of Sensitivity}

Various efforts have been made for the enhancement of sensitivity of fiber optic SPR sensors. First step towards the enhancement of sensitivity was the tapering of optical fiber probes [44, 45]. Single and dual tapered optical fiber SPR sensors have also been reported [46, 47]. The effect of various taper profiles on the performance of an optical fiber SPR sensor was studied for the optimization of the performance [48]. It was concluded that the sensitivity of the sensor is maximum when the taper profile is exponential linear. Further, a U- shaped fiber optic SPR sensor was theoretically studied for the enhancement of sensitivity [49]. In both the 


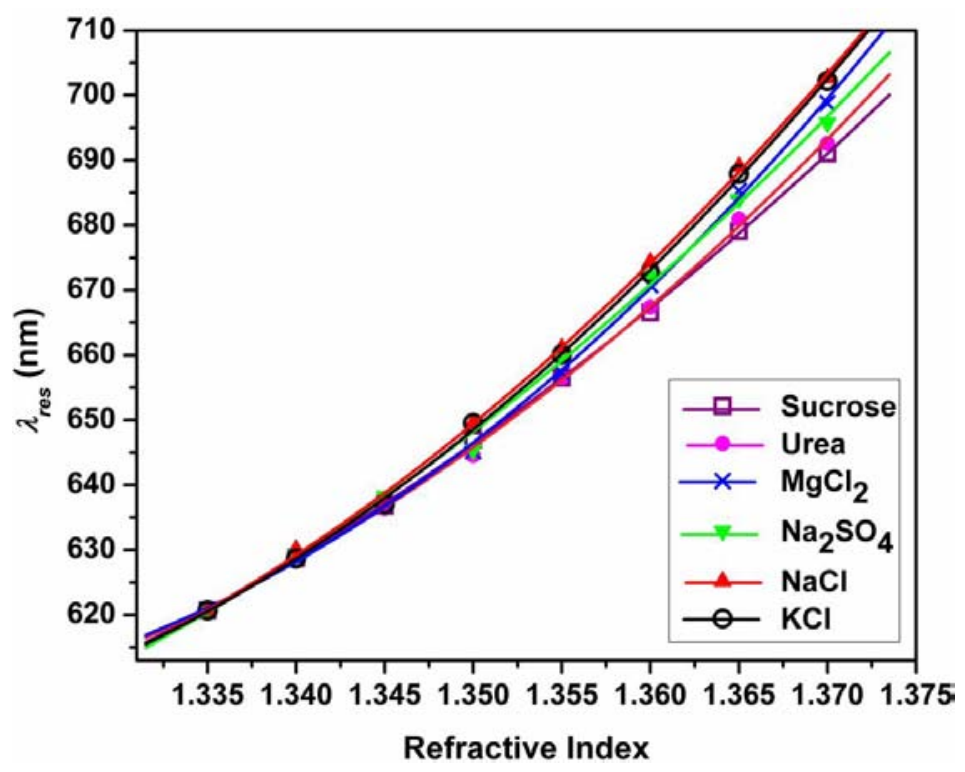

Fig. (15). Variation of resonance wavelength $\left(\lambda_{\text {res }}\right)$ with the refractive index of sucrose, urea, $\mathrm{Na}_{2} \mathrm{SO}_{4}, \mathrm{MgCl}_{2}, \mathrm{NaCl}$ and $\mathrm{KCl}$ solutions (Reprinted with permission from Elsevier [43]).

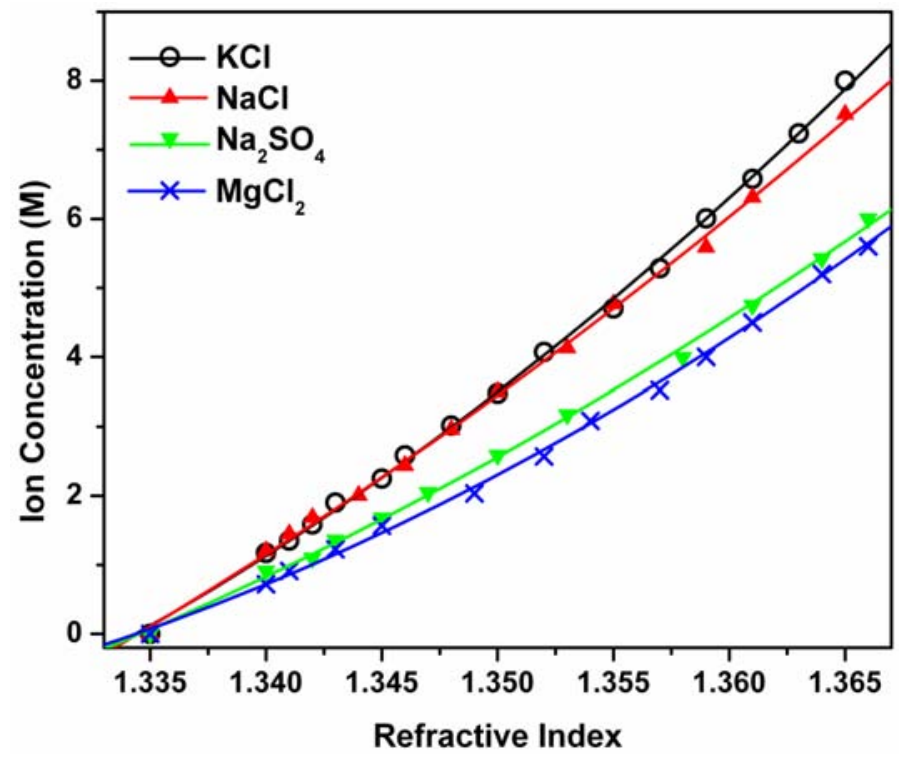

Fig. (16). Variation of ion concentration with the refractive index of sucrose, urea, $\mathrm{Na}_{2} \mathrm{SO}_{4}, \mathrm{MgCl}_{2}, \mathrm{NaCl}$ and $\mathrm{KCl}$ solutions. (Reprinted with permission from Elsevier [43]).

cases, the tapering and U-shaped probes, an enhancement in the strength of coupling of the evanescent field with surface plasmons takes place, which is responsible for the increase in the sensitivity. However, these sensors further suffer the disadvantage of fragility and reduced detection accuracy, as the SPR curves broaden with tapering. To provide robustness to the tapered fiber probes, multi-tapered fiber optic SPR sensors were prepared [50]. In such probes, the taper ratio (ratio of the untapered diameter to the taper waist) was kept close to unity and multiple tapers were written in the same sensing length, shown in Fig. (17). The distance between two tapered regions was termed as taper period $(\Lambda)$. Fig. (18) shows the variation of the resonance wavelength with refractive index for different taper periods [50]. It was observed that with an increase in the number of tapers the resonance wavelength showed a red shift, while the total shift in resonance wavelength was also increased. The red shift in resonance wavelength was attributed to the fact that tapering leads to an increase in the penetration depth. Since the penetration depth is proportional to the wavelength, the coupling of light at higher wavelengths becomes stronger, in turn red shifting the resonance wavelength. Further, the tapering increases the number of reflections within the sensing length, which increases the sensitivity, but also decreases the detection accuracy.

Few researchers reported fiber optic plasmonic sensors with a very thin layer of silicon over metal film [51]. These sensors work on the principle of guided wave plasmonics and possess better sensitivity than usual fiber optic plasmonic sensors. Fiber optic sensors utilizing guided wave 


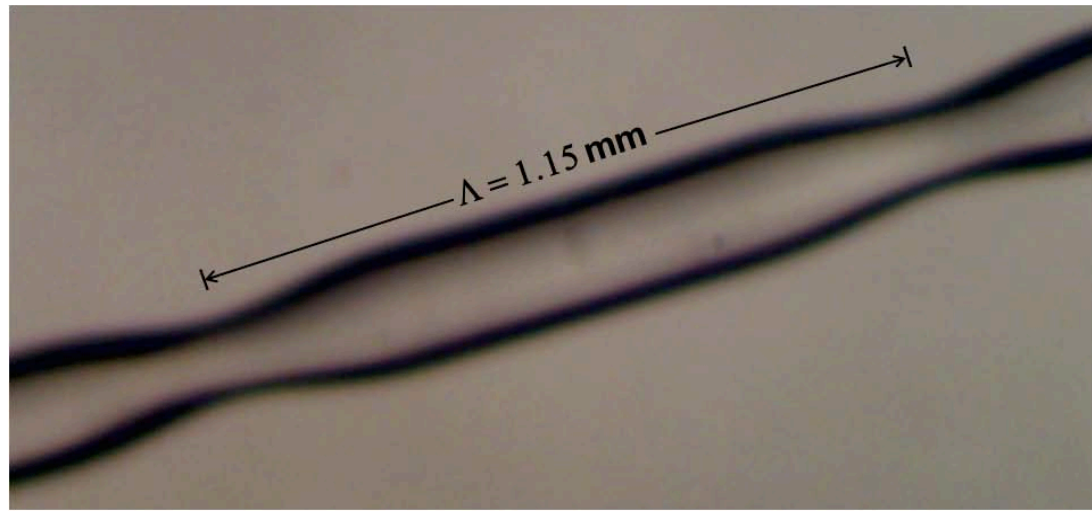

Fig. (17). Microscopic view of the tapered optical fiber core (Reprinted with permission from IEEE [50]).

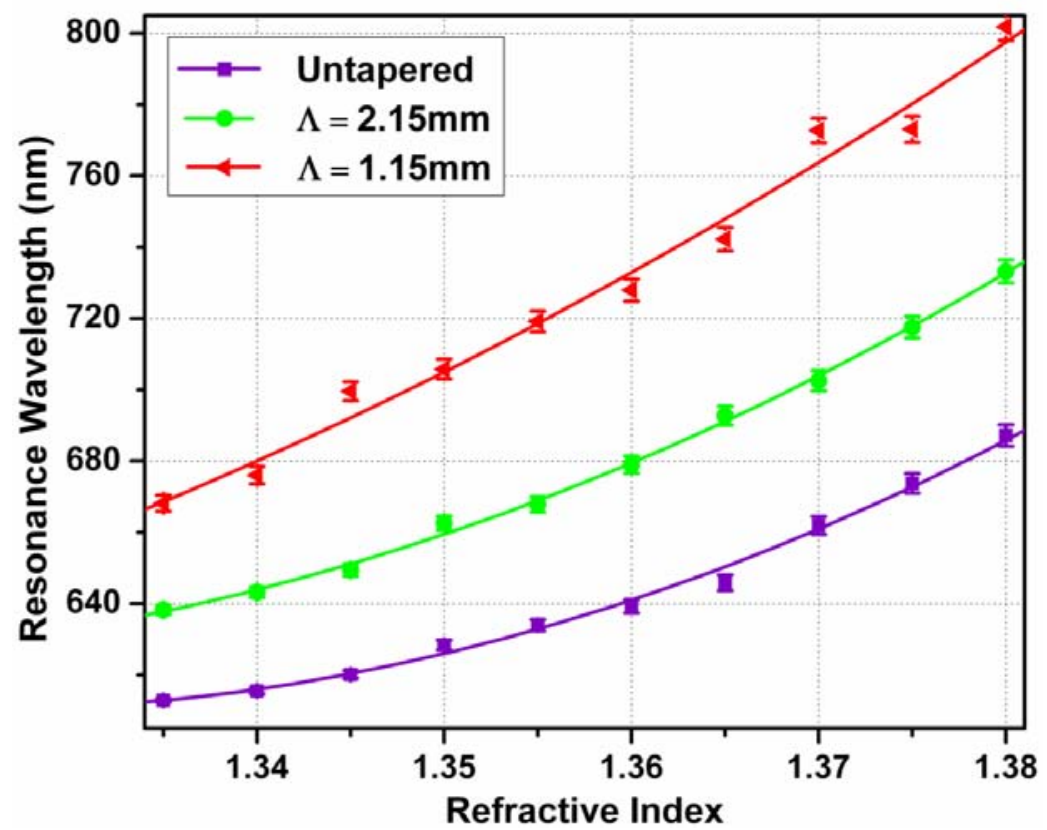

Fig. (18). Variation of $\lambda_{\text {res }}$ with refractive index the probes with different $\Lambda$ (Reprinted with permission from IEEE [50]).

plasmonics have also been fabricated for the detection of urea [52].

\section{LOCALIZED SURFACE PLASMONS}

\subsection{Brief History}

The field of localized surface plasmons is much older than that of propagating ones. The gold nanoparticles are known from ancient times and were originally used in staining glasses. The 'soluble' gold appeared probably in the $4^{\text {th }}$ or $5^{\text {th }}$ century B.C. in China and Egypt [53]. The Lycurgus Cup in the British museum, made by the Romans in the fourth century $\mathrm{AD}$, appears green in the reflected light (in daylight). However, when viewed in light transmitted through the glass, it appears red. The red color is due to small particles of metallic gold [54]. Ancient stained glass makers knew that by putting very tiny amounts of gold and silver in glass they could produce red and yellow colors. By placing small amounts of tiny nanoparticles in some particular fashion, the physical properties of a material can be changed. In the fifteenth and sixteenth centuries, the potters started using glazes containing copper and silver nanoparti- cles. In the sixteenth century (about 1548), Cipriano Piccolpasso provided the recipe for the nanoparticulate glazes in his book 'Li tre libri dell'arte del vasaiol'. He used a mixture of copper and silver salts with vinegar, ochre, and clay and applied these mixtures to the surface of already glazed pottery [55].

The reputation of gold sols until the middle ages was to explore its fabulous curative powers for various diseases, such as heart and venereal problems, epilepsy, dysentery and tumors and for the diagnosis of syphilis. The colloidal gold sols were called aurum potabile (drinkable gold) by the alchemists of the Middle Ages. The first book on colloidal gold was 'Panacea Aurea-Auro Potabile', written by Francisci Antonii in 1618. This book provides considerable information about the preparation of gold sols and their uses in medical applications, including some practical cases [56]. In 1676, a German chemist, Johann Kunckels, published a book named 'Nuetaliche Observationes oder Anmerkungen von Auro und Argento potabili' which described the then recent observations on gold and silver sols and their applications [57]. In 1685, Andreas Cassius published a treatise ' $D e$ 


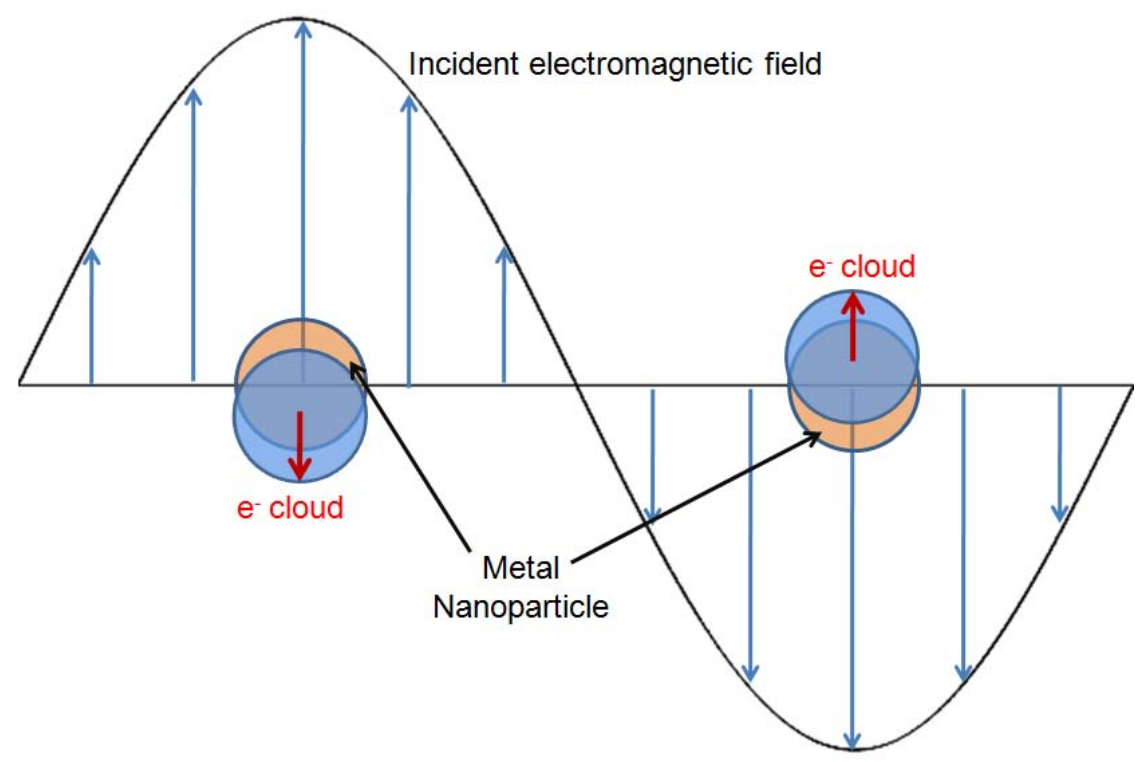

Fig. (19). Schematic of a spherical metal nanoparticle under the action of a rapidly varying field.

Auro', which provided the recipe for making the colouring pigment or stain by gold [58]. Since then the colour has been called 'Purple of Cassius'. A complete treatise on colloidal gold was written by Hans Heinrich Helchar in the beginning of the eighteenth century [59]. In this treatise, he stated that the use of boiled starch in its drinkable gold preparation noticeably enhanced its stability. In 1794, Fulhame wrote a book which reported that silk could be dyed by colloidal gold [60]. In 1818, Jeremias Bejamin Richters explained the differences in colors shown by gold sols prepared by different methods [61]. He pointed out that the pink or purple solutions contain gold in the finest degree of subdivision, whereas the yellow solutions result due to the formation of aggregates of the particles. Faradey, in 1857, reported the formation of deep red colloidal gold solutions by the reduction of an aqueous solution of tetrachloroaurate using phosphorus in $\mathrm{CS}_{2}$ [62]. He investigated the optical properties of thin films of dried colloidal solutions and observed reversible color changes upon mechanical compression. This was probably the first use of metal nanoparticles for any sensing application (pressure). Around 1897, almost 250 years after the discovery of Purple of Cassius, Richard Zsigmondy showed that it consisted of colloidal gold and stannic acid. He was awarded the Nobel Prize in Chemistry in 1925. In the 20th century, various methods for the preparation of gold colloids were reported and reviewed. Turkevich et al. (1951) reported the preparation of colloidal gold particles by reduction of aqueous solution of tetrachloroauric acid by sodium citrate [63]. The method was modified by many research groups to obtain particles of uniform size and shape. The theoretical analysis of small nanostructures started in 1908, when Gustav Mie developed a theory of scattering and absorption exhibited by small colloidal particles of gold suspended in water [64]. Around the same time, Peter Debye solved the problem of radiation pressure exerted on small particles in free space, one of the first applications of Mie theory to the astrophysical problem. Since then various attempts have been made by Kreibig and others to study the optical properties of small metal nanostructures due to the changes in their electronic properties at such a small level [65].

\subsection{General Overview}

Localized surface plasmons are the quanta of charge density oscillations in metal nanoparticles. They get excited when the frequency of the incident light becomes equal to that of oscillation frequency of conduction electrons of the metal nanoparticle. Fig. (19) shows the schematic of the action of a varying electric field on a spherical metal nanoparticle. It can be seen in Fig. (19) that the conduction electron cloud of the nanoparticle gets shifted in a direction opposite to the direction of the electric field vector of the incident field, leaving behind the positive background. Thus, the overall positive and negative charge centers of the nanoparticles get displaced depending upon the strength of the electric field and thus behave like a dipole. Under the action of rapid variation of incident electric field $\left(\sim 10^{14} / \mathrm{sec}\right)$, this dipole starts oscillating. At a certain incident frequency, the polarizability of the nanoparticle shows enhanced resonant oscillations. This results in the absorbance of incident light and is called localized surface plasmon (LSP) resonance. The plasmon resonances of metal nanoparticles are characterized by their extinction cross-section, which is the sum of its scattering and absorption cross-sections. The extinction crosssection is highly dependent on the shape, size, material and environment of the metal nanoparticle [66].

The dependence of the LSP resonance wavelength on the refractive index of the nanoparticle is generally utilized for the sensing applications. Fig. (20) shows the LSPR spectra for a spherical gold nanoparticle of $5 \mathrm{~nm}$ radius surrounded by three different dielectric media. As the refractive index of the medium surrounding $\left(\sqrt{\varepsilon_{s}}\right)$ the nanoparticle increases, the resonance wavelength gets red shifted. By measuring the change in the resonance wavelength, one can predict the 


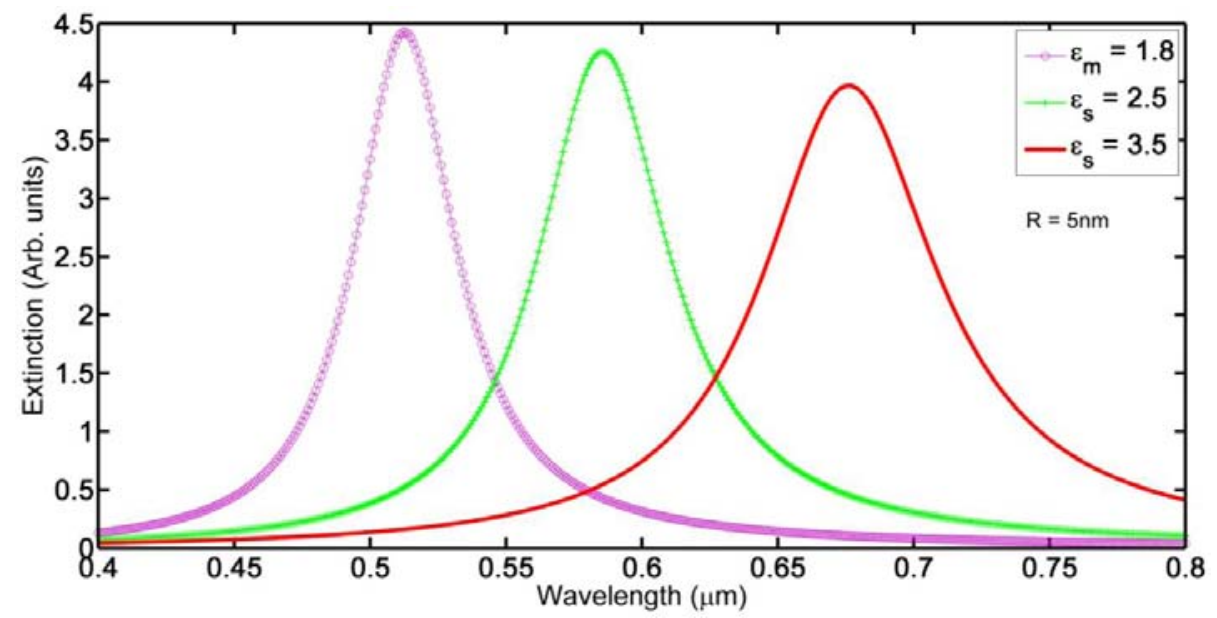

Fig. (20). Simulated LSPR spectra for different dielectric constants of the surrounding medium.

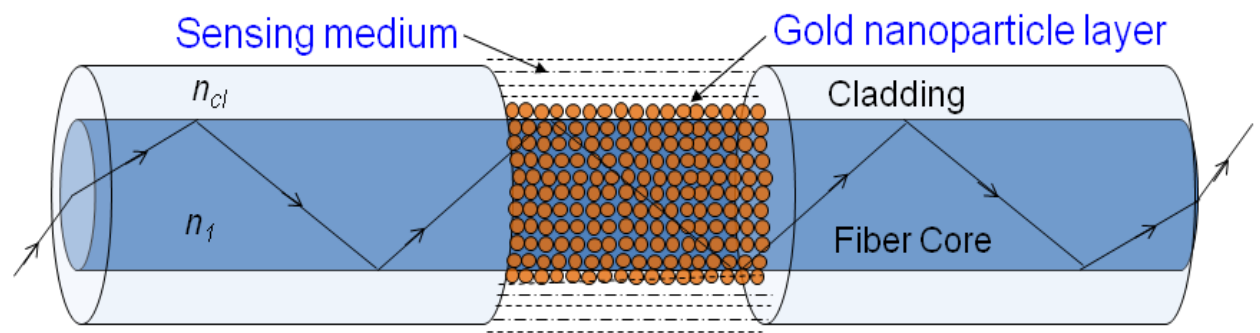

Fig. (21). Schematic of a fiber optic LSPR sensor.

change in refractive index value. Metal nanoparticles of various shapes and sizes have been utilized by many researchers for sensing applications [66].

\subsection{LSPR Based Fiber Optic Refractive Index Sensor}

Fiber optic LSPR sensors have got attention due to their compact size and low cost. The most general scheme of such a sensor is shown in Fig. (21). In such a sensor, a small portion of the cladding is removed from the middle of the optical fiber and a monolayer of the metal nanoparticle is coated over the unclad portion. The sensing medium further surrounds the nanoparticle coated region. The evanescent field of the light guided inside the core of the optical fiber excites the plasmon resonance of the metal nanoparticles. As a result, a small portion of the guided spectral range gets absorbed by the nanoparticle layer and a peak is observed in the absorption spectrum of transmitted optical power. First experiment on such sensor was carried out by Chau et al. for biosensing applications [67]. Lin et al reported LSPR based fiber optic reflection probe for chemical sensing at high pressure conditions [68]. Later they presented a LSPR based $\mathrm{Cd}$ sensor [69]. Around the same time, Sai et al studied LSPR sensors for immunoglobulin- anti immunoglobulin (IgG-anti IgG) interactions [70]. We now present below some studies on LSPR based fiber optic sensors.

\section{(a) Refractive Index Sensor}

Simulations were carried out to study a fiber optic LSPR based refractive index sensor [71]. The response of the sensor was studied for various light launching conditions and light sources such as collimated monochromatic and polychromatic source and diffuse source like LEDs. Figs. (22) and (23) show the variation of effective evanescent coefficient per nanoparticle with dielectric constant for a diffuse source such as light emitting diode (LED) having Gaussian power profile [71]. The sensor response in this case is studied for intensity modulation scheme of operation. It was observed that with an increase in the dielectric constant of the surrounding medium, the effective evanescent absorbance per nanoparticle increases, reaches to a maximum at certain dielectric constant value and then again starts decreasing. This can be understood as follows: A change in the dielectric constant of the sensing medium changes the position of the peak of the LSPR spectrum of the nanoparticle. This leads to the change in overlap of the LSPR spectrum with the profile of the light source. Since, the LSPR spectrum shows a red shift with an increase in the sensing medium dielectric constant, the overlap of the LSPR spectrum first increases with increase in dielectric constant, reaches to the maximum overlap, and then starts decreasing with further increase in the dielectric constant, as the LSPR spectrum now moves away from the source bandwidth region.

\section{(b) Temperature Sensor}

A LSPR based fiber optic temperature sensor having gold nanoparticles in the core of the optical fiber was realized by Dhawan and Muth [72]. The sensitivity of the sensor was, however, low. Şayan and Özdemir [73] used $\mathrm{TiO}_{2}$ in a prism based SPR sensor while Sharma and Gupta [74] used it in fiber optic SPR sensor for temperature sensing. The simulations were performed to use LSPR for fiber optic temperature sensing [75]. Fig. (24) shows the schematic design of the simulated fiber optic LSPR probe. The probe consists of spherical gold nanoparticles uniformly dispersed in a sensing 


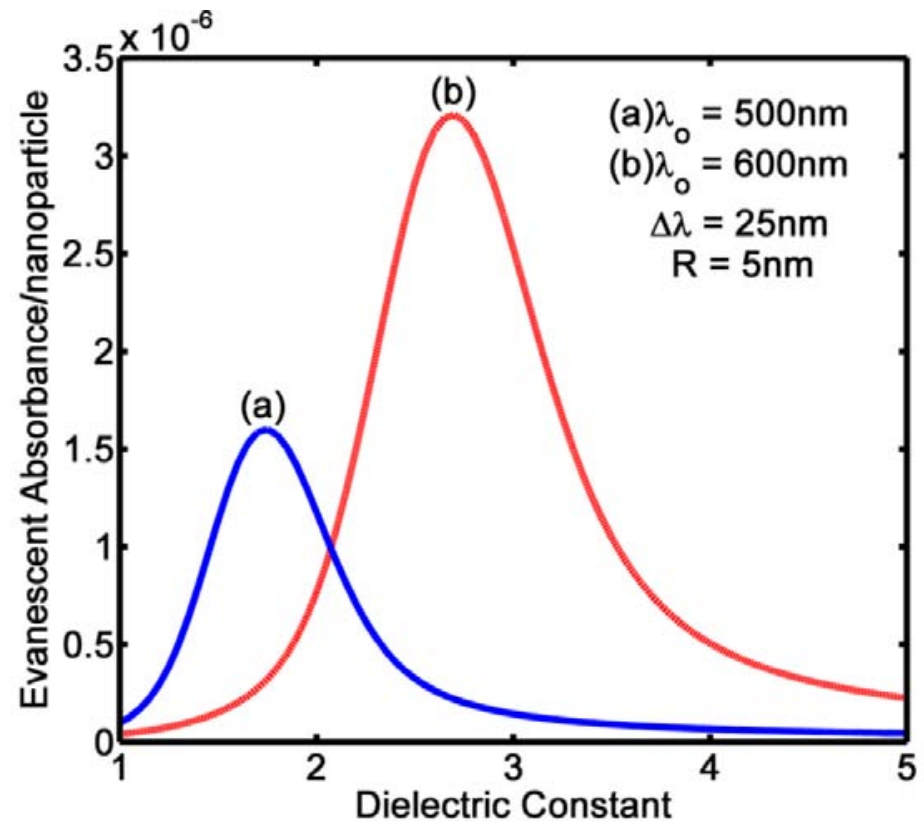

Fig. (22). Variation of evanescent absorbance with dielectric constant of the sensing medium for two different diffuse sources. (Reprinted with permission from OSA [71]).

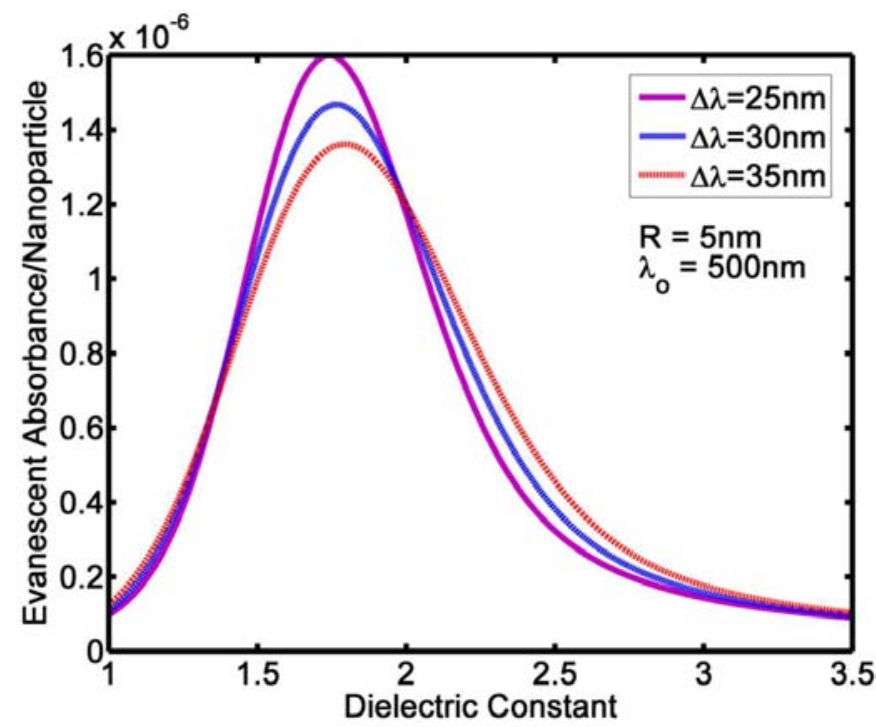

Fig. (23). Variation of effective evanescent absorbance with dielectric constant of the sensing medium for different source bandwidths (Reprinted with permission from OSA [71]).

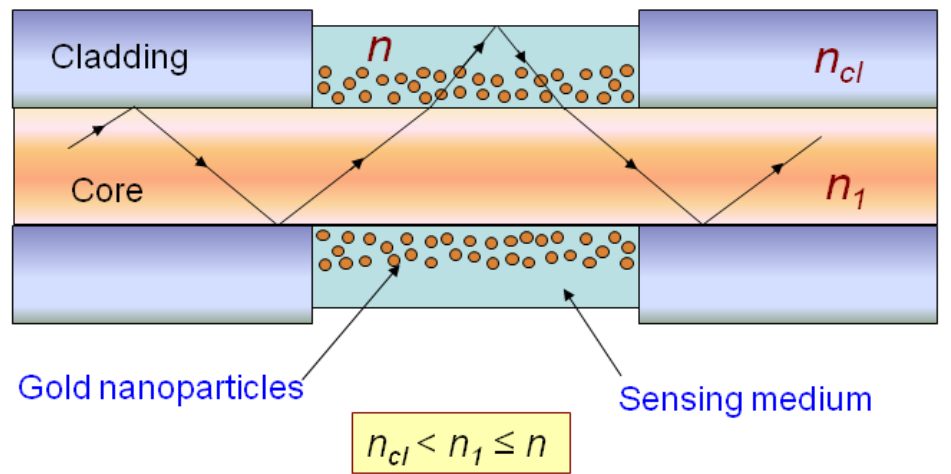

Fig. (24). Schematic of the proposed fiber optic temperature probe utilizing metal nanoparticles. (Reprinted with permission from OSA [75]). 


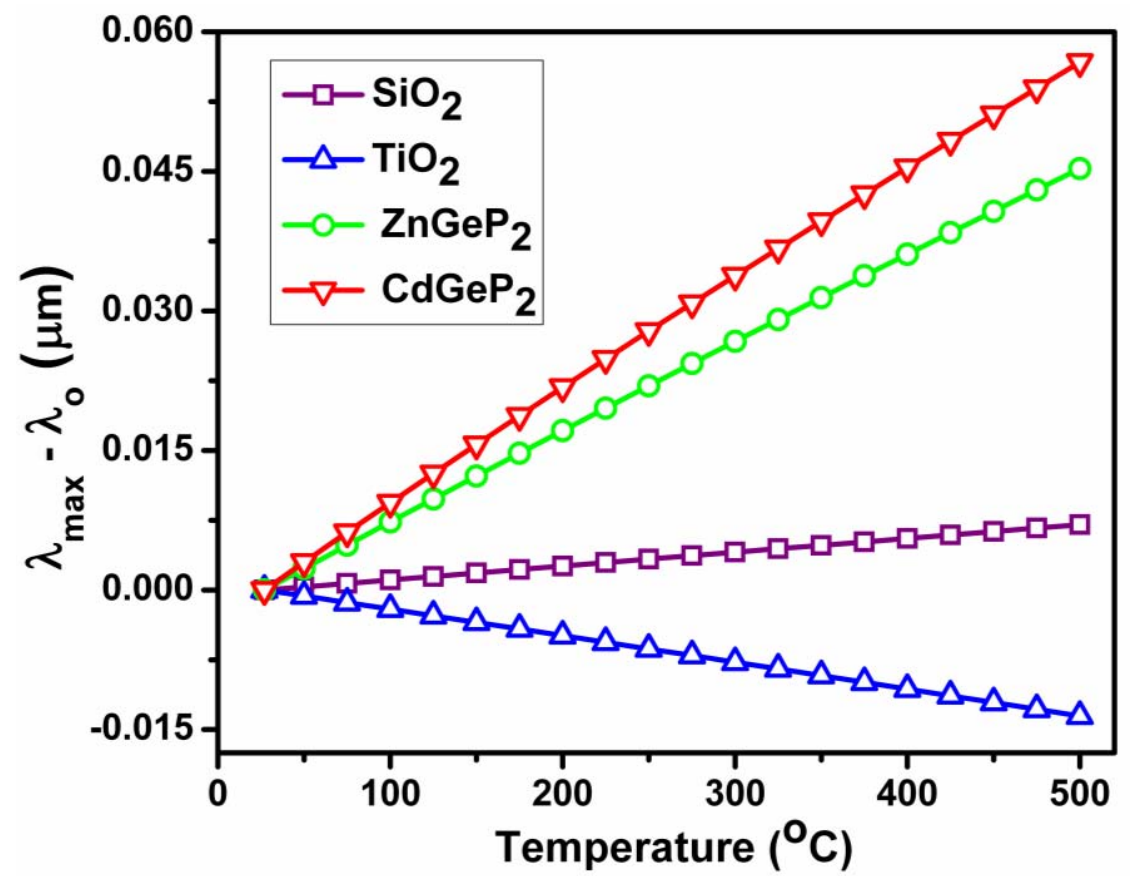

Fig. (25). Variation of difference in peak resonance wavelengths $\left(\lambda_{\max }-\lambda_{\mathrm{o}}\right)$ with temperature for $\mathrm{SiO}_{2}, \mathrm{TiO}_{2}, \mathrm{ZnGeP}_{2}$ and $\mathrm{CdGeP}{ }_{2}$ as surrounding media. $\lambda_{\mathrm{o}}$ is the corresponding peak resonance wavelength at $27^{\circ} \mathrm{C}$. (Reprinted with permission from OSA [75]).

medium, which is coated on the unclad portion of the optical fiber. The refractive index of the sensing medium was kept greater than that of core and cladding. In such case, the light guided in the optical fiber gets refracted at the core-sensing medium interface, excites localized surface plasmons, reaches the sensing medium-air interface, from where it gets total internally reflected and then comes back to the core. The sensor works on spectral interrogation method. The simulations were carried out for four different dielectric media $\left(\mathrm{SiO}_{2}, \mathrm{TiO}_{2}, \mathrm{ZnGeP}\right.$ and $\left.\mathrm{CdGeP}_{2}\right)$ as sensing medium. $\mathrm{SiO}_{2}$ and $\mathrm{TiO}_{2}$ were considered as sensing media because these have already been used in various temperature sensing applications [72-74], while the temperature sensing properties of $\mathrm{ZnGeP}_{2}$ and $\mathrm{CdGeP}_{2}$ were explored for the first time in the study. The thermo-optic coefficients of these materials are high. The resonance wavelengths calculated from the simulated LSPR spectra for the four sensing media were plotted with temperature and are shown in Fig. (25). It was observed that the change in resonance wavelength with temperature is the maximum in considered materials. The sensitivity of the sensor, calculated from the slope of curve shown in Fig. (25), is tabulated in Table 1. It was found that the sensitivity of the sensor with $\mathrm{CdGeP}_{2}$ as sensing medium is 10 times greater than $\mathrm{SiO}_{2}$ and $\mathrm{TiO}_{2}$, which are conventional materials used for temperature sensing. Though $\mathrm{TiO}_{2}$ has a high thermo-optic coefficient, it being negative, cancels the effect of temperature on the shift in LSPR wavelength.

\section{(c) Hydrogen Gas Sensor}

The plasmon resonances of palladium (Pd) nanoparticles were used for fiber optic hydrogen gas $\left(\mathrm{H}_{2}\right)$ sensor by Prabhakar et al. [76]. Palladium is a good absorber of $\mathrm{H}_{2}$ and it is widely used for $\mathrm{H}_{2}$ sensing applications. But, as discussed above in earlier cases, it has always been used as a sensing medium. However, the sensor fabricated by Prabhakar and co-workers works on the principle of change in the dielectric constant of the metal nanoparticle by absorption of $\mathrm{H}_{2}$.

Table 1. Sensitivity of the Sensor for Different Dielectric Materials. (Reprinted with Permission from OSA [75])

\begin{tabular}{|c|c|}
\hline Sensing Material & Sensitivity $\left(\mu \mathbf{m} /{ }^{\circ} \mathbf{C}\right)$ \\
\hline \hline $\mathrm{SiO}_{2}$ & $1.485 \times 10^{-5}$ \\
\hline $\mathrm{TiO}_{2}$ & $-2.875 \times 10^{-5}$ \\
\hline $\mathrm{ZnGeP}_{2}$ & $9.495 \times 10^{-5}$ \\
\hline $\mathrm{CdGeP}_{2}$ & $1.184 \times 10^{-4}$ \\
\hline
\end{tabular}

\subsection{Influence of Temperature}

It has already been established that the performance of a SPR sensor gets affected by any change in the ambient temperature [77]. However, it is highly demanded that the performance of a sensor does not change with any fluctuation in temperature. So, the performance of a fiber optic LSPR sensor was studied, both theoretically and experimentally, under the changing ambient temperature conditions [78]. Further, the plasma and collision frequencies of metal nanoparticle are temperature dependent. This leads to a change in the real and imaginary parts of the dielectric constant of the metal nanoparticle with a change in ambient temperature. Fig. (26) shows the wavelength dependent variation of (a) real $\left(\varepsilon_{1}\right)$ and (b) imaginary $\left(\varepsilon_{2}\right)$ parts of the dielectric constant of a spherical metal nanoparticle of $5 \mathrm{~nm}$ radius at different temperatures. It is observed that both $\varepsilon_{1}$ and $\varepsilon_{2}$ increase with an increase in temperature. Since the real part of the dielectric constant increases with temperature, a red shift in the LSPR spectrum will occur with increase in temperature. 

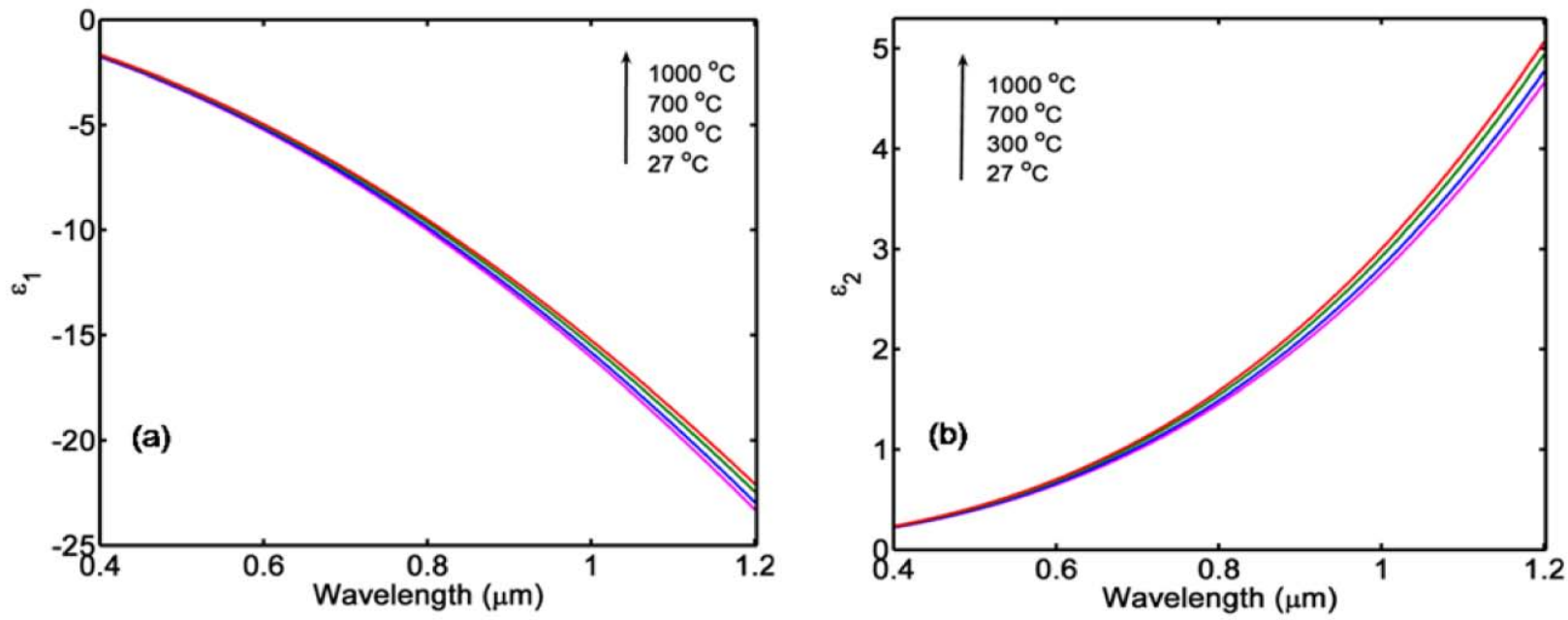

Fig. (26). Variation of the dielectric constant of the spherical metal nanoparticle of radius $5 \mathrm{~nm}$ with wavelength at different temperatures. (a) real part, and (b) imaginary part. (Reprinted with permission from OSA [75]).

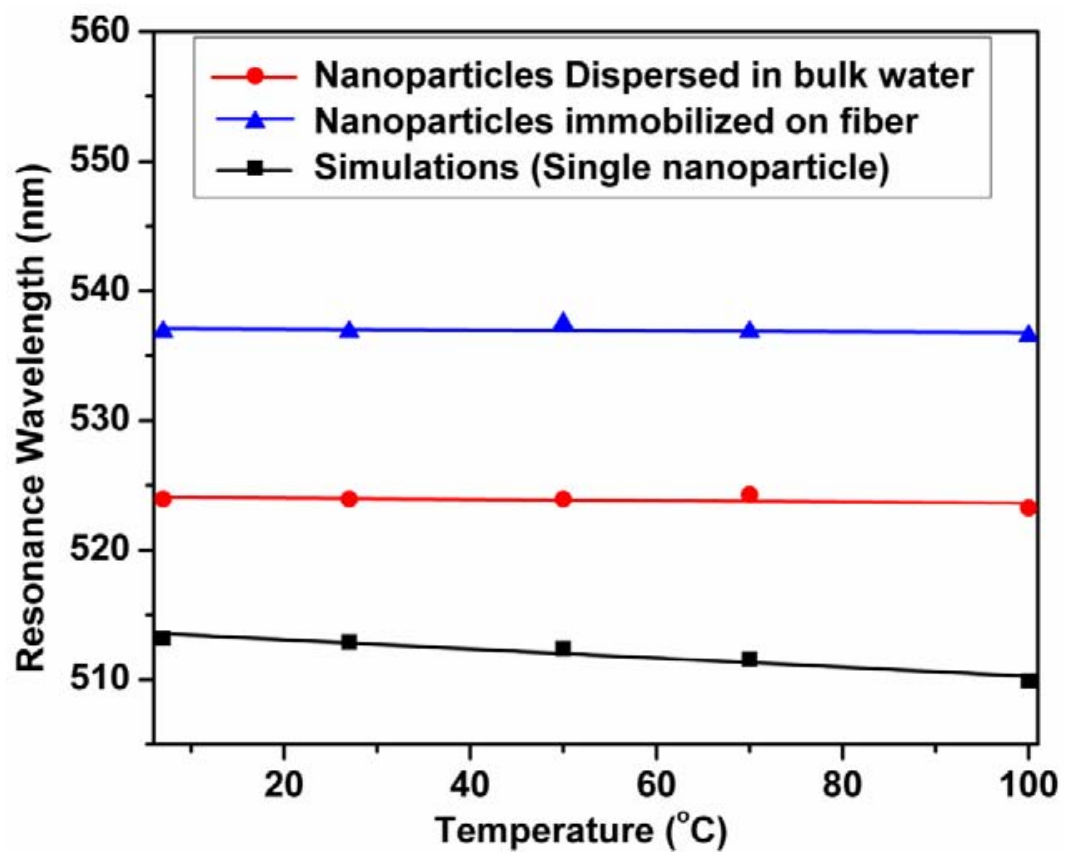

Fig. (27). Experimental and simulated results for the variation of resonance wavelength with the temperature of the water as the sensing medium. ((Reprinted with permission from IFSA [78])).

The temperature dependence of the dielectric functions of optical fiber core, metal nanoparticle and sensing medium was considered for simulations. Water was used as sensing medium because it is the mostly used solvent and is a medium for almost all the biological entities. The characteristics of the sensor reported have been plotted in Fig. (27) and (28), both in (a) wavelength and (b) intensity modulation scheme of operation. The numerical results have been calculated by considering only single nanoparticle. From both the plots, it was concluded that in spectral interrogation scheme of operation, the performance of the sensor remains nearly unaffected, as only $3 \mathrm{~nm}$ shift in resonance wavelength was observed for a temperature change of $7{ }^{\circ} \mathrm{C}$ to $100{ }^{\circ} \mathrm{C}$; while in intensity modulation scheme, the performance of the sensor got affected and a calibration factor depending upon temperature must be included in the characteristic curve.

\subsection{Enhancement of Performance}

The sensitivity of an optical fiber evanescent wave absorption sensor has been found to increase either by tapering or by making a $\mathrm{U}$ shaped probe $[46,79]$. In both the cases, the evanescent field of the guided light extends more in the sensing region, leading to increased absorbance, and hence sensitivity. In the case of fiber optic LSPR sensors, it has been reported in literature that the intensity modulation mode of operation is more sensitive than the spectral interrogation scheme. Hence, by making U-shaped probes, the absorbance of light by metal nanoparticles can be enhanced. The LSPR based U-shaped fiber optic sensor was fabricated for studying the interaction between IgG- anti IgG [70]. The LSPR based U-shaped fiber optic sensor provides the further advantage of point sensing, which leads to miniaturization of sensing sample volume. A U-shaped fiber optic LSPR sensor 


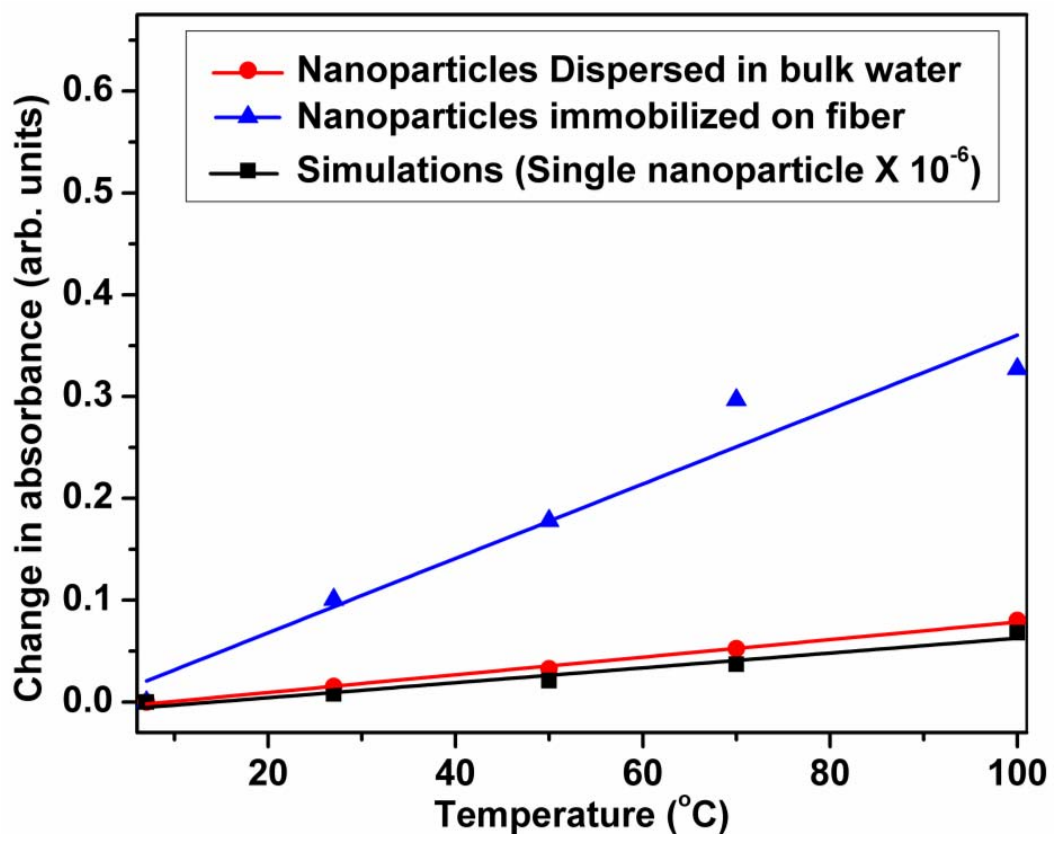

Fig. (28). Variation of difference in absorbance $\left(\mathrm{A}_{\mathrm{t}}-\mathrm{A}_{7}\right)$ at any temperature with reference to $7{ }^{\circ} \mathrm{C}$ with temperature (Reprinted with permission from IFSA [78]).

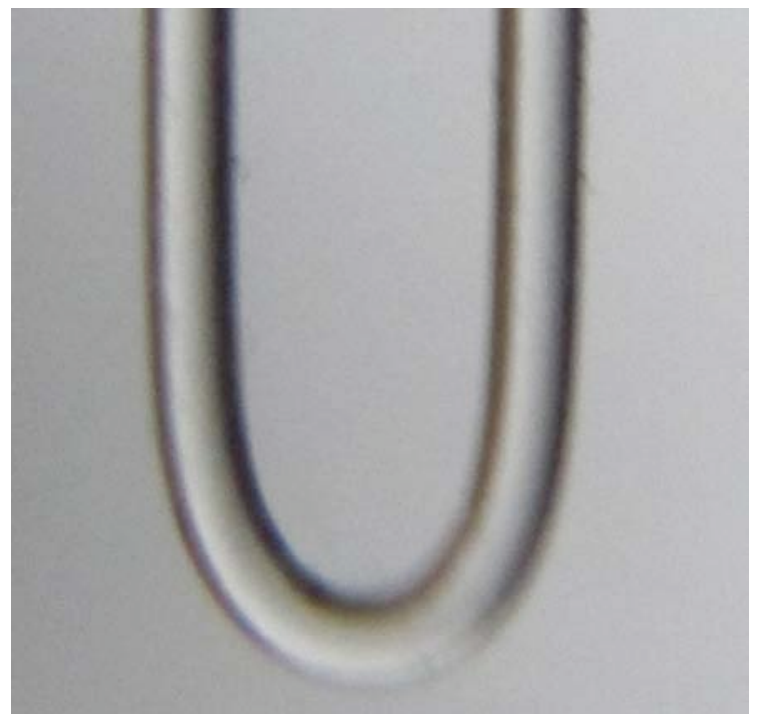

Fig. (29). Microscopic image of the prepared U-shaped optical fiber probe (Reprinted with permission from Springer [80]).

was fabricated for the detection of blood glucose [80]. Fig. (29) shows the microscopic view of a U-bent optical fiber. Such a probe was first immobilized with gold nanoparticles and then by glucose oxidase by chemical binding. The schematic of the experimental setup used for the characterization of the sensor is shown in Fig. (30). Light launched from a polychromatic source was fed into the optical fiber, in which the evanescent field excites LSPR and then was collected off the output end by a spectrometer interfaced with a computer. Fig. (31) shows the LSPR spectra recorded for different concentrations of aqueous solutions of glucose varying from 0 $\mathrm{mg} / \mathrm{dl}$ to $250 \mathrm{mg} / \mathrm{dl}$. This range of glucose concentration was chosen to mimic the human blood glucose concentrations. It was observed that as the glucose concentration increases, the LSPR absorbance decreases. However, a negligible shift in the resonance wavelength with increase in the glucose con- centration was observed. The reason behind the negligible shift in resonance wavelength was reported to be due to the negligible change in the refractive index of the sample solutions in the considered concentration range. The change in absorbance occurs due a small change in the local refractive index of glucose oxidase because of the following reaction

Glucose $+\mathrm{O}_{2} \stackrel{\text { Glucose Oxidase }}{\longrightarrow}$ GluconicAcid $+\mathrm{H}_{2} \mathrm{O}_{2}$

As the concentration of glucose in the vicinity of the sensing region increases, the change in the local refractive index increases which leads to larger change in the absorbance. The decrease in absorbance with glucose concentration can easily be observed in the inset. The response of the sensor changes with change in the bending radius of the $\mathrm{U}$ shaped probe. This happens due to the change in penetration 


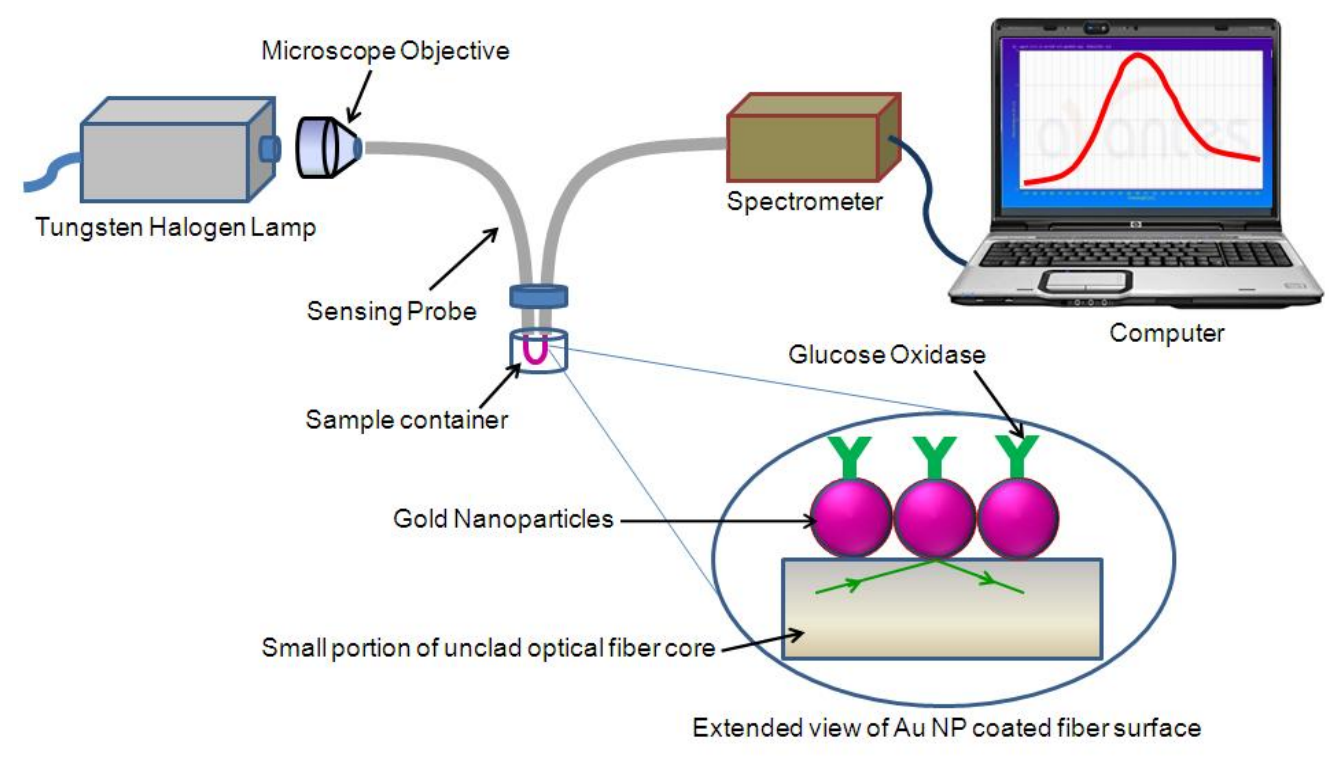

Fig. (30). Schematic experimaental set-up for the characterization of U-shape LSPR (Reprinted with permission from Springer [80]).

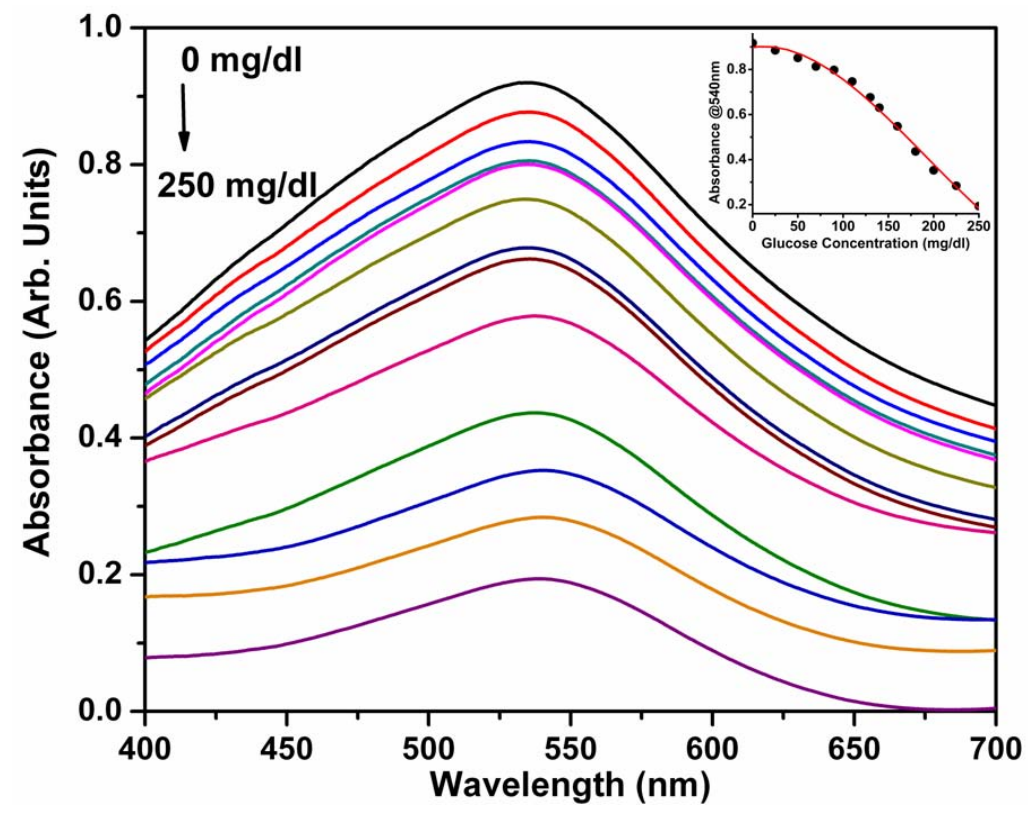

Fig. (31). Absorbance spectra of U- shaped LSPR probe of bending radius $0.711 \mathrm{~mm}$ for different concentrations of glucose. (Reprinted with permission from Springer [80]).

depth in the case of different bending radii. The sensors with different bending radii were fabricated for the optimization of the performance of the sensor. The LSPR spectra were recorded for all the sensors and the sensitivity was evaluated from them. The variation of sensitivity with the bending radius is shown in Fig. (32) for the fixed glucose concentration of $100 \mathrm{mg} / \mathrm{dl}$. It was observed that, for a fixed glucose concentration, the sensitivity of the sensor increases with decrease in the bending radius, reaches a maximum value for certain bending and then again starts decreasing with further bending. The reason behind such a variation can be understood as follows: As we start bending an optical fiber, the evanescent field starts penetrating more to the sensing region, which, in turn, leads to an increase in the strength of coupling between the light and localized surface plasmons. This happens up to a certain bending radius. For further increase in the bending (i.e. decrease in the bending radius), the light starts leaking in to the outer medium, which is responsible for the decrease in the sensitivity. The sensitivity of a U-shaped fiber optic LSPR sensor was obtained to be maximum around $1 \mathrm{~mm}$ bending radius.

\section{THE FUTURE}

Optical fiber SPR sensors for multianalyte and multichannel sensing have been realized recently. A rigorous theoretical and experimental study of such sensor was performed recently by Verma et al. [81]. In such a sensor, dif- 


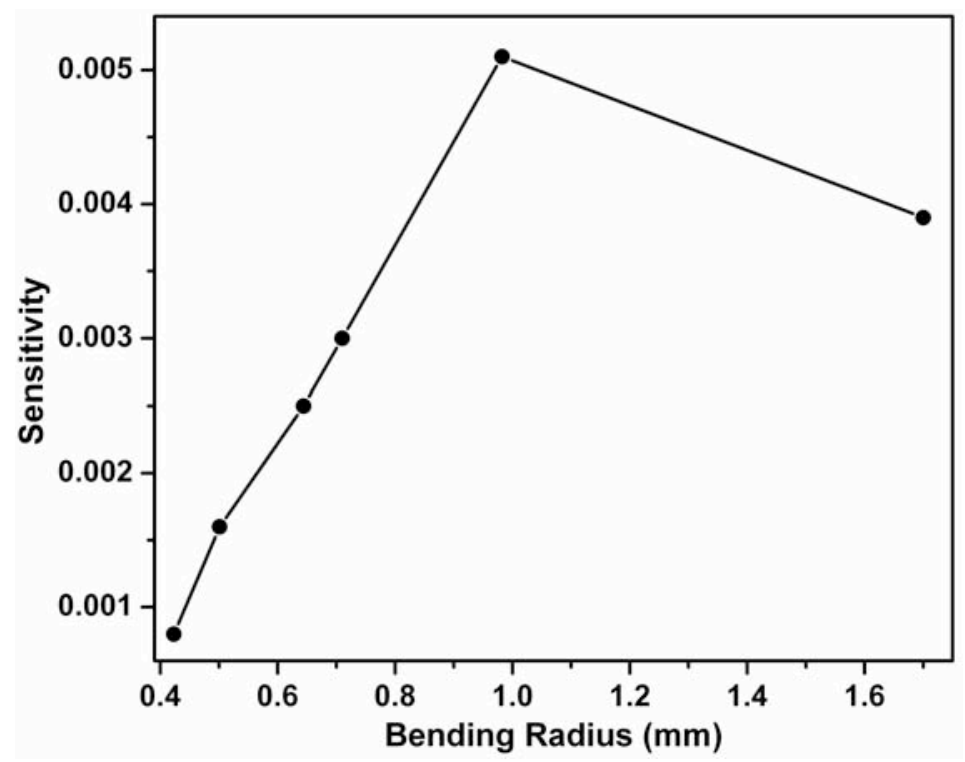

Fig. (32). Variation of sensitivity with bending radius at the glucose concentration $100 \mathrm{mg} / \mathrm{dl}$ (Reprinted with permission from Springer [80]).

ferent regions of an optical fiber with different plasmonic materials can be utilized for simultaneous sensing of multiple analytes. The sensor uses the different spectral regions of the visible light spectrum. It is anticipated that, in coming future, lot of applications of multichannel sensing may come to realization of sensing of multiple analytes. The simultaneous collaboration of SPR and LSPR in the same optical fiber sensing probe is also foreseen. The plasmonic resonances have now reached to the detection limit of single molecules [6]. People have now started fabricating novel plasmonic structures for sensing applications [82]. These sensing structures can be collaborated simultaneously for sensing of multiple parameters. The recent attempts have been towards fabricating photonic sensor chips for fast and reliable detection of biological species [83]. However, these sensors chips can be of much benefit if collaborated with optical fiber [84]. They can then be used for online and fast detection, remote sensing and multi-parameter sensing. Plasmonic sensor chips with optical fiber integration seem to be future sensor devices. Apart from that, some recent reports show a huge enhancement of performance of various nanophotonic sensors and whispering gallery mode (WGM) resonators [85].The whispering gallery modes are generally excited by the evanescent field of tapered optical fibers. The WGM sensors have been found to have very low detection limit, up to single molecules. When collaborated with plasmonic structures, they are seen to detect single molecule interactions more accurately and precisely [86].

\section{APPENDIX A}

\section{Surface Plasmons: Mathematical Framework}

Surface plasmons are the modes supported by a metaldielectric interface. These are basically surface waves, which get generated by the cumulative action of conduction electron oscillations and incident electromagnetic field. These waves travel along the metal-dielectric interface and their field amplitude decays exponentially in both the media. As shown in Fig. (1), if there be a metal-dielectric interface along the $\mathrm{z}$-direction, then the SP wave propagates along the $\mathrm{x}$ axis. A complete treatise about surface plasmons can be found in ref. [87]. However, we present below a brief and simple mathematical framework of surface plasmons. Let the refractive index of a dielectric medium be $\varepsilon_{s}$ and that of the metal be $\varepsilon_{m}=\varepsilon_{1}+i \varepsilon_{2}$, where $\varepsilon_{1}$ and $\varepsilon_{2}$ are respectively the real and imaginary parts of the metal dielectric function. The electric and magnetic field components of the four Maxwell's equations are written and then the boundary conditions are satisfied to obtain the modal fields. From the modal fields supported by the interface, the expressions for propagation constant, propagation length and penetration depth are deduced. The Maxwell's equations are given as:

$$
\begin{aligned}
& \nabla \cdot \vec{D}=\rho_{\text {ext }} \\
& \nabla \cdot \vec{B}=0 \\
& \nabla \times \vec{E}=-\frac{\partial \vec{B}}{\partial t} \\
& \nabla \times \vec{H}=\vec{J}_{e x t}+\frac{\partial \vec{D}}{\partial t}
\end{aligned}
$$

where $\vec{D}, \vec{B}, \vec{E}$ and $\vec{H}$ represent the four macroscopic fields: dielectric displacement, magnetic induction, electric field and magnetic field respectively; $\rho_{\text {ext }}$ and $\vec{J}_{e x t}$ denote the external charge and current densities respectively.

For time varying harmonic fields $(\partial / \partial t=-i \omega)$, the Maxwell's equations can be rearranged to give two sets of self-consistent solutions having different polarizations. One set of solutions is called the transverse electric (TE or s) mode and other set transverse magnetic (TM or p). For the kind of geometry shown in Fig. (1), the set of $\left(H_{x}, E_{y}\right.$ and $\left.H_{z}\right)$ corresponds to TE mode and the set of $\left(E_{x}, H_{y}\right.$ and $\left.E_{z}\right)$ corresponds to TM mode. The corresponding TE and TM wave equations are given by equations (A.5) and (A.6). 


$$
\begin{aligned}
& \frac{d^{2} E_{y}}{d z^{2}}+\left(k_{o}^{2} n^{2}-\beta^{2}\right) E_{y}=0 \\
& \frac{d^{2} H_{y}}{d z^{2}}+\left(k_{o}^{2} n^{2}-\beta^{2}\right) H_{y}=0
\end{aligned}
$$

where $k_{o}\left(=2 \pi / \lambda_{o}\right)$ is the wave vector of the incident electromagnetic wave in free space and $\beta$ is the wave vector of the propagating wave in the medium. The solutions with evanescent decay in the z-direction are only considered because the propagating wave solutions confined to a metal-dielectric interface are only of interest.

Decaying wave solutions of (A.5) and (A.6) can be given as,

For $z>0, E_{y}=A e^{-\sqrt{\beta^{2}-k_{o}^{2} \varepsilon_{s}} z}$

$H_{y}=B e^{-\sqrt{\beta^{2}-k_{o}^{2} \varepsilon_{s}} z}$

For $z<0, E_{y}=C e^{\sqrt{\beta^{2}-k_{o}^{2} \varepsilon_{m}} z}$

$H_{y}=D e^{\sqrt{\beta^{2}-k_{o}^{2} \varepsilon_{m}} z}$

The boundary conditions for TE waves are given as

(a) $\left.E_{y}\right|_{\text {boundary }}=$ continuous and (b) $\left.\frac{\partial E_{y}}{\partial z}\right|_{\text {boundary }}=$ continuous .

Applying the boundary condition (a) from equations (A.7) and (A.9), one gets

$A=C$

Use of this in the boundary condition (b), yields

$-A \sqrt{\beta^{2}-k_{o}^{2} \varepsilon_{s}}=A \sqrt{\beta^{2}-k_{o}^{2} \varepsilon_{m}}$

Squaring both the sides and rearranging the terms, one obtains

$$
A k_{o}^{2}\left(\varepsilon_{s}-\varepsilon_{m}\right)=0
$$

Since $\varepsilon_{s}$ and $\varepsilon_{m}$ are of opposite signs (neglecting the imaginary part), the term in brackets cannot be zero. Also, for the term in the bracket to be zero, both the terms must individually be zero. This implies that $A=0$. Thus no surface modes can exist for a TE polarized light.

Similarly, applying the boundary conditions for TM modes, eqs. (A.8) and (A.10) yield

$$
\begin{aligned}
& \frac{-\sqrt{\beta-k_{o}^{2} \varepsilon_{s}}}{\varepsilon_{s}}=\frac{\sqrt{\beta-k_{o}^{2} \varepsilon_{m}}}{\varepsilon_{m}} \\
& \frac{\beta-k_{o}^{2} \varepsilon_{s}}{\varepsilon_{s}^{2}}=\frac{\beta-k_{o}^{2} \varepsilon_{m}}{\varepsilon_{m}^{2}} \\
& \beta^{2}\left(\varepsilon_{s}^{2}-\varepsilon_{m}^{2}\right)=k_{o}^{2} \varepsilon_{s} \varepsilon_{m}\left(\varepsilon_{s}-\varepsilon_{m}\right)
\end{aligned}
$$

$$
\begin{aligned}
& \beta^{2}=\frac{k_{o}^{2} \varepsilon_{s} \varepsilon_{m}\left(\varepsilon_{s}-\varepsilon_{m}\right)}{\left(\varepsilon_{s}-\varepsilon_{m}\right)\left(\varepsilon_{s}+\varepsilon_{m}\right)} \\
& \beta=k_{o} \sqrt{\frac{\varepsilon_{s} \varepsilon_{m}}{\left(\varepsilon_{s}+\varepsilon_{m}\right)}}
\end{aligned}
$$

This is the expression for the wave vector of surface plasmons supported by the metal-dielectric interface.

Thus, $k_{s p}=\beta=k_{o} \sqrt{\frac{\varepsilon_{s} \varepsilon_{m}}{\left(\varepsilon_{s}+\varepsilon_{m}\right)}}$

The field associated with the surface plasma wave can be given as

$E=E_{o} \exp \left[+i\left(k_{x} x \pm k_{z} z-\omega t\right)\right]$

Where, + and - signs are for $z \geq 0$ and $z \leq 0$, respectively and the propagation constant $k_{z}$ is imaginary which signifies the exponential decay of the field (Fig. (2)). The wave vector $k_{x}$ lies parallel to the $\mathrm{x}$ direction and is given by $k_{x}=\beta$. There are two important parameters associated with surface plasmon waves; propagation length and penetration depth. These are presented below.

\section{A.1. Propagation Length}

Surface plasmon wave is a decaying wave. It decays, exponentially, both into the metal as well as in the dielectric medium. In Eq.(2.3), the dielectric constant of the metal, $\varepsilon_{m}$, is a complex number and therefore the propagation constant of the surface plasmon wave $k_{s p}$ will also be a complex number having real and imaginary parts i.e.

$k_{s p}=k_{x}=k_{x}^{\prime}+i k_{x}^{\prime \prime}$

$k_{x}^{\prime}=\frac{\omega}{c}\left(\frac{\varepsilon_{m}^{\prime} \varepsilon_{d}}{\varepsilon_{m}^{\prime}+\varepsilon_{d}}\right)^{1 / 2}$

$k_{x}^{\prime \prime}=\frac{\omega}{c}\left(\frac{\varepsilon_{m}^{\prime} \varepsilon_{d}}{\varepsilon_{m}^{\prime}+\varepsilon_{d}}\right)^{1 / 2} \frac{\varepsilon_{m}^{\prime \prime}}{2\left(\varepsilon_{m}^{\prime}\right)^{2}}$

$\varepsilon_{m}=\varepsilon_{1}+i \varepsilon_{2}$ is the dielectric constant of the metal with $\varepsilon_{1}$ and $\varepsilon_{2}$ as the real and imaginary parts. Therefore, the intensity of the surface plasmon wave propagating along the interface decreases as $e^{-2 k_{x}^{\prime \prime} x}$. The length over which the intensity of the surface plasmon wave decreases to 1/e of its maximum value is called the propagation length and is given by [87]

$L_{s p}=\left(2 k_{x}^{\prime \prime}\right)^{-1}$ 
Table A.1. Values of Propagation Length for Gold and Silver Interfaced with Air at 543 and 633 nm

\begin{tabular}{|c|c|c|}
\hline \multicolumn{2}{|c|}{ Propagation Length } \\
\hline & at $\lambda=\mathbf{5 4 3} \mathbf{~ n m}$ & at $\lambda=\mathbf{6 3 3} \mathbf{~ n m}$ \\
\hline \hline Gold & $10.17 \mu \mathrm{m}$ & $15.34 \mu \mathrm{m}$ \\
\hline Silver & $29.82 \mu \mathrm{m}$ & $43.67 \mu \mathrm{m}$ \\
\hline
\end{tabular}

Table A.2. Values of Penetration Depth at $\lambda=543 \mathrm{~nm}$ and $633 \mathrm{~nm}$

\begin{tabular}{|c|c|c|c|c|}
\hline \multirow{2}{*}{} & \multicolumn{2}{|c|}{ Penetration Depth } & \multicolumn{2}{c|}{ at $\lambda=\mathbf{6 3 3} \mathbf{~ n m}$} \\
\cline { 2 - 5 } & Metal $\lambda=\mathbf{5 4 3} \mathbf{~ n m}$ & Dielectric & Metal & Dielectric \\
\hline \hline \multirow{2}{*}{ Gold } & $26.67 \mathrm{~nm}$ & $250.12 \mathrm{~nm}$ & $26.76 \mathrm{~nm}$ & $350.18 \mathrm{~nm}$ \\
\hline Silver & $23.08 \mathrm{~nm}$ & $298.52 \mathrm{~nm}$ & $23.12 \mathrm{~nm}$ & $414.48 \mathrm{~nm}$ \\
\hline
\end{tabular}

The values of propagation length for surface plasmons calculated for gold and silver interfaced with air at two wavelengths $543 \mathrm{~nm}$ and $633 \mathrm{~nm}$ of $\mathrm{He}-\mathrm{Ne}$ laser have been tabulated below in Table A.1.

\section{A.2. Penetration Depth}

The propagation constant of a wave along the $\mathrm{z}$ direction can be given by [87]

$k_{z i}=\left\{\beta^{2}-\varepsilon_{i}\left(\frac{\omega}{c}\right)^{2}\right\}^{1 / 2}$

$i=1,2$ correspond to metal and dielectric media respectively.

The field amplitude of surface plasmon wave decreases exponentially as $e^{\left(-\left|k_{z i}\right||z|\right)}$ perpendicular to the interface. The distance over which the field amplitude falls to 1/e of its value at the surface is called the penetration depth $(\delta)$ of the surface plasmon wave.

The values of the penetration depth for calculated silverair and gold-air interface at $\lambda=543 \mathrm{~nm}$ and $633 \mathrm{~nm}$ are tabulated in Table A.2.

\section{APPENDIX B}

\section{Localized Surface Plasmons: Mathematical Formulation}

Localized surface plasmons are the non-propagating excitations of conduction electrons of metallic nanostructures coupled to an electromagnetic field [88]. These modes are the solutions of the scattering problem of a small, subwavelength conductive nanoparticle in an oscillating electromagnetic field. In order to derive the expression for the resonance condition, the interaction of a small metal nanoparticle with an electromagnetic wave is considered first. The interaction of a particle of diameter $D$ with the electromagnetic field can be analyzed by using a simple quasi-static approximation, provided that the particle size is much smaller than the wavelength of incident light, i.e. $D<<$ $\lambda$. In such an approximation, the phase of the oscillating electromagnetic field experienced by the nanoparticle remains approximately constant over its volume. So the problem reduces to the calculation of spatial field distribution for a small metal particle in an electrostatic field. The harmonic time dependence of the electric field is added only after the field distributions have been obtained. A concise but clear understanding of derivation of localized surface plasmon extinction can be found in ref. [88]; however, ref. [89] presents a rigorous derivation of the extinction cross-section.

Let there be a homogeneous, isotropic metal nanosphere of radius $\mathrm{R}$ put at the origin in a uniform, static electric field $\vec{E}=E_{o} \hat{z}$. The medium surrounding the nanoparticle is considered to be isotropic and non-absorbing, with a dielectric constant $\varepsilon_{s}$. The lines of electric field are considered to be parallel to the $\mathrm{z}$ axis. A schematic of a spherical metal nanoparticle put in a uniform electric field is shown in Fig. (B1).

In electrostatic approach, generally the potentials $\Phi$ are first obtained by solving the Laplace equation $\nabla^{2} \Phi=0$, and then electric fields $\vec{E}$ are calculated by finding the gradient of the potential as $\vec{E}=-\nabla \Phi$. Due to azimuthal symmetry of the present problem, general solution of the Laplace equation can be written as [90],

$\Phi(r, \theta)=\sum_{l=0}^{\infty}\left[A_{l} r^{l}+B_{l} r^{-(l+1)}\right] P_{l}(\cos \theta)$

where $P_{l}(\cos \theta)$ are the Legendre Polynomials of order $l$, and $\theta$ is the angle between the position vector $\vec{r}$ at the point $\mathrm{P}$ and the $\mathrm{z}$-axis (Fig. (B1)). Since the potential at the origin has to remain finite, the potentials inside $\left(\Phi_{\text {in }}\right)$ and outside $\left(\Phi_{\text {out }}\right.$ ) the sphere can be written as

$\Phi_{i n}(r, \theta)=\sum_{l=0}^{\infty} A_{l} r^{l} P_{l}(\cos \theta)$ 


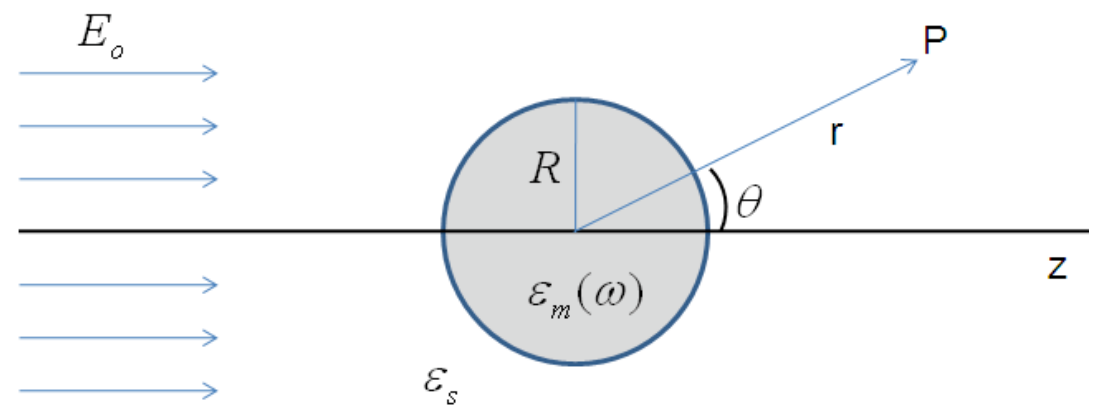

Fig. (B1). A schematic of a sphere in a uniform electric field.

\section{Scattered}

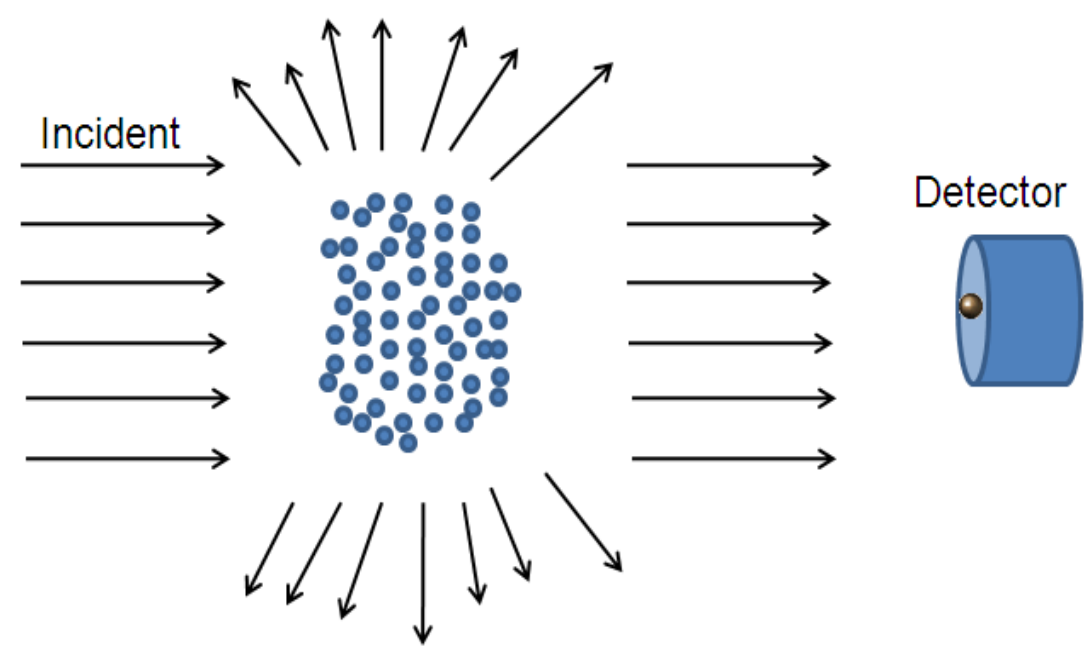

Fig. (B2). Extinction by an ensemble of particles.

$\Phi_{\text {out }}(r, \theta)=\sum_{l=0}^{\infty}\left[B_{l} r^{l}+C_{l} r^{-(l+1)}\right] P_{l}(\cos \theta)$

At the boundary of the sphere and surrounding medium (i.e. at $r=R$ ) the potentials must satisfy $\Phi_{\text {in }}=\Phi_{\text {out }}$ and $\varepsilon_{m} \frac{\partial \Phi_{\text {in }}}{\partial r}=\varepsilon_{s} \frac{\partial \Phi_{\text {out }}}{\partial r}$. Applying these boundary conditions, one gets

$-\left.\frac{1}{R} \frac{\partial \Phi_{\text {in }}}{\partial \theta}\right|_{r=R}=-\left.\frac{1}{R} \frac{\partial \Phi_{\text {out }}}{\partial \theta}\right|_{r=R}$

and $-\left.\varepsilon_{o} \varepsilon_{m} \frac{\partial \Phi_{i n}}{\partial r}\right|_{r=R}=-\left.\varepsilon_{o} \varepsilon_{s} \frac{\partial \Phi_{o u t}}{\partial r}\right|_{r=R}$

From (B.4) and (B.5), we get $A_{l}=C_{l}=0$ for $l \neq 1$. Also, at large distances from the sphere, the electric field remains the same as unperturbed incident field. This implies that

$\lim _{r \rightarrow \infty} \Phi_{\text {out }}=-E_{o} z=-E_{o} r \cos \theta$

which demands that $B_{1}=-E_{o}$ and $B_{l}=0$ for $l \neq 1$. Calculating the values of the coefficients $A_{l}$ and $C_{l}$ and putting the values of all the coefficients in eqs. (B.2) and (B.3), one gets
$\Phi_{i n}=-\frac{3 \varepsilon_{s}}{\varepsilon_{m}+\varepsilon_{s}} E_{o} r \cos \theta$

$\Phi_{\text {out }}=-E_{o} r \cos \theta+\frac{\varepsilon_{m}-\varepsilon_{s}}{\varepsilon_{m}+2 \varepsilon_{s}} E_{o} R^{3} \frac{\cos \theta}{r^{2}}$

Equation (B.8) shows that $\Phi_{\text {out }}$ is a superposition of potentials due to the incident field and that due to a dipole located at the center of the nanoparticle. In terms of dipole moment $\vec{p}$, eq. (B.8) can be rewritten as

$\Phi_{\text {out }}=-E_{o} r \cos \theta+\frac{\vec{p} \cdot \vec{r}}{4 \pi \varepsilon_{o} \varepsilon_{s} r^{3}}$

where $\vec{p}=4 \pi \varepsilon_{o} \varepsilon_{s} R^{3} \frac{\varepsilon_{m}-\varepsilon_{s}}{\varepsilon_{m}+2 \varepsilon_{s}} \vec{E}_{o}$

Equation (B.10) implies that the incident field induces a dipole moment inside the nanosphere and the magnitude of this induced dipole moment is proportional to the magnitude of the strength of the incident field. For a sphere, the dipole moment can be rewritten in terms of polarizability $(\alpha)$ as

$\vec{p}=\varepsilon_{o} \varepsilon_{s} \alpha \vec{E}_{o}$

Thus 
$\alpha=4 \pi R^{3} \frac{\varepsilon_{m}-\varepsilon_{s}}{\varepsilon_{m}+2 \varepsilon_{s}}$

From eq. (B.12), it can be concluded that the polarizability experiences a resonant enhancement when $\left|\varepsilon_{m}+2 \varepsilon_{s}\right|$ becomes a minimum. For small values of $\varepsilon_{2}$, the resonance condition can be written as

$\varepsilon_{1}=-2 \varepsilon_{s}$

This relation is named as the Frölich condition. The associated mode is called the surface plasmon mode (dipole) of the metal nanoparticle. The spatial distribution of the electric field inside and outside the sphere is given by the relation $\vec{E}=-\nabla \Phi$. From eqs. (B.7) and (B.9) one gets

$\vec{E}_{i n}=\frac{3 \varepsilon_{s}}{\varepsilon_{m}+2 \varepsilon_{s}} \vec{E}_{o}$

$\vec{E}_{\text {out }}=\vec{E}_{o}+\frac{3 \vec{n}(\vec{n} \cdot \vec{p})-\vec{p}}{4 \pi \varepsilon_{o} \varepsilon_{s} R^{3}}$

After calculating the field distributions due to an electrostatic field, the time variance of the electric field can be imposed now. Under the illumination of a plane wave $\vec{E}(\vec{r}, t)=\vec{E}_{o} e^{-i \omega t}$, the induced dipole moment is given as (from (B.11))

$\vec{p}(t)=\varepsilon_{o} \varepsilon_{s} \alpha \vec{E}_{o} e^{-i \omega t}$

The radiation of this dipole is responsible for the scattering of the plane wave by the sphere, which can be represented as the radiation by a point dipole sitting at the centre of the sphere. For the purpose of calculation of the fields, the sphere can be replaced with an ideal dipole located at $z=0$. The total fields $\vec{E}(t)=\vec{E} e^{-i \omega t}$ and $\vec{H}(t)=\vec{H} e^{-i \omega t}$ in the near, intermediate and radiation zones of a dipole can be written as

$\vec{E}=\frac{1}{4 \pi \varepsilon_{o} \varepsilon_{s}}\left[k^{2}(\vec{n} \times \vec{p}) \times \vec{n} \frac{e^{i k r}}{r}+\{3 \vec{n}(\vec{n} \cdot \vec{p})-\vec{p}\}\left(\frac{1}{r^{3}}-\frac{i k}{r^{2}}\right) e^{i k r}\right]$

and $\vec{H}=\frac{c k^{2}}{4 \pi}(\vec{n} \times \vec{p}) \frac{e^{i k r}}{r}\left(1-\frac{1}{i k r}\right)$

where $k=2 \pi / \lambda$ and $\vec{n}$ is the unit vector in the direction of point $\mathrm{P}$.

Since the magnitude of the magnetic field is about a fac-

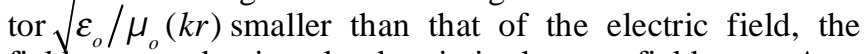
fields are predominantly electric in the near field zone. As a consequence of the enhancement of polarization due to resonance, a tremendous enhancement in efficiency of scattering and absorption of light by the metal nanoparticle occurs. The corresponding cross-sections for scattering $\left(C_{s c a}\right)$ and absorption $\left(C_{a b s}\right)$ can be calculated via Poynting vector, as it has the dimensions of energy per unit area and time. After obtaining the electromagnetic fields inside and outside the particle, one can determine the Poynting vector at any point. However, in the present case, the Poynting vectors at points outside the particle are of great interest. The time averaged Poynting vector $\vec{S}$ at any point in the medium surrounding the nanoparticle can be written as

$\vec{S}=\frac{1}{2} \operatorname{Re}\left\{\vec{E} \times \vec{H}^{*}\right\}=\vec{S}_{i}+\vec{S}_{s}+\vec{S}_{\text {ext }}$

where $\vec{S}_{i}=\frac{1}{2} \operatorname{Re}\left\{\vec{E}_{i} \times \vec{H}_{i}^{*}\right\}$

$\vec{S}_{s}=\frac{1}{2} \operatorname{Re}\left\{\vec{E}_{s} \times \vec{H}_{s}^{*}\right\}$ and $\vec{S}_{e x t}=\frac{1}{2} \operatorname{Re}\left\{\vec{E}_{i} \times \vec{H}_{s}^{*}+\vec{E}_{s} \times \vec{H}_{i}^{*}\right\} ; \vec{S}_{i}$ and $\vec{S}_{s}$ being the Poynting vectors associated with the incident and scattered fields respectively. $\vec{S}_{e x t}$ is the term arising due to the interaction between the scattered and incident fields.

Let us consider an ensemble of nanoparticles exposed to a beam of electromagnetic radiation with power $U_{o}$ (Fig. (B2)). If the power received by the detector be $U$, then $U<U_{o}$. The presence of nanoparticles results in the extinction of the incident beam. If the medium surrounding the nanoparticles be non-absorbing, then the difference in power $U_{o}-U$ accounts for the absorption in the nanoparticles (i.e. transformation of electromagnetic energy into other forms) and scattering by the nanoparticles. The extinction depends on the chemical composition, size, shape and orientation of nanoparticles, refractive index of the surrounding medium, number of particles and the state of polarization and frequency of the incident light.

Let us consider extinction by a single nanoparticle embedded in a non-absorbing medium. Let the nanoparticle be illuminated by a plane wave (Fig. B3). We construct an imaginary sphere of radius $r$ around the particle. The net rate at which electromagnetic energy crosses the surface $A$ of the sphere is

$W=-\int_{A} S . \hat{r} d A$

$W$ consists of contributions from incident $\left(W_{i}\right)$, scattered $\left(W_{\text {sca }}\right)$ and extinction $\left(W_{\text {ext }}\right)$ rates as follows:

$W=W_{i}-W_{s c a}+W_{e x t}$

where $W_{i}=-\int_{A} \vec{S}_{i} . \hat{r} d A, W_{s c a}=-\int_{A} \vec{S}_{s} \cdot \hat{r} d A$ and $W_{e x t}=-\int_{A} \vec{S}_{e x t} . \hat{r} d A$.

$W_{i}$ vanishes identically as the surrounding medium is non-absorbing. Since the medium surrounding the nanoparticle is non-absorbing, $W$ is the rate at which energy is absorbed by the nanoparticle, i.e. $W=W_{a b s}$. Therefore, $W_{e x t}=W_{s c a}+W_{a b s}$.

If $I_{i}$ denotes the incident energy per unit area and time $\left(\left|\vec{S}_{i}\right|\right)$, then the ratios of $W_{s c a}, W_{a b s}$ and $W_{e x t}$ to $I_{i}$ lead to quantities having the dimensions of area. These quantities are called the scattering $\left(C_{s c a}\right)$, absorption $\left(C_{a b s}\right)$ and extinction $\left(C_{e x t}\right)$ cross-sections respectively and denoted as: 


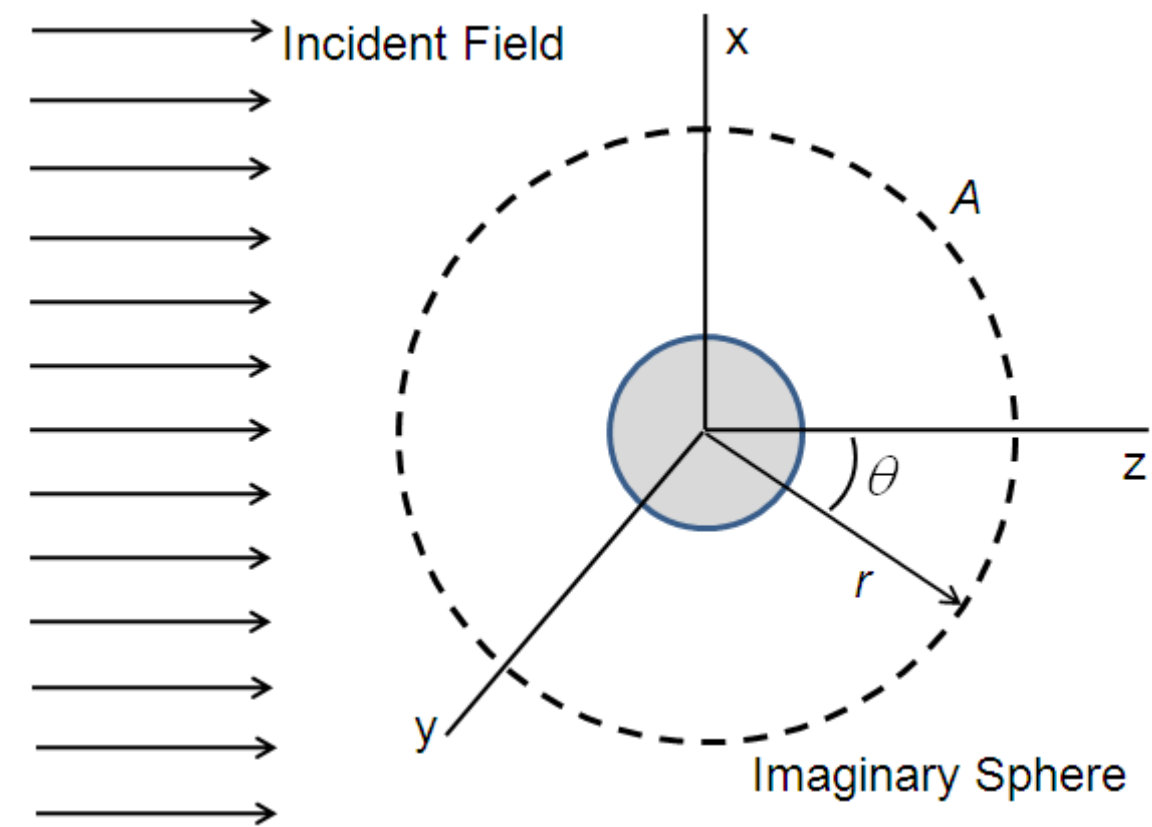

Fig. (B3). Extinction by a single particle.

$C_{s c a}=\frac{W_{s c a}}{I_{i}}, C_{a b s}=\frac{W_{a b s}}{I_{i}}$ and $C_{e x t}=\frac{W_{e x t}}{I_{i}}$

Solving for the $W_{s c a}, W_{a b s}$ and $W_{e x t}$ values by calculating the amplitude scattering matrices, substituting in eq. (B22) and after a bit of algebra, we get

$$
\begin{aligned}
& C_{s c a}=\frac{k^{4}}{6 \pi}|\alpha|^{2}=\frac{8 \pi}{3} k^{4} R^{6}\left|\frac{\varepsilon_{m}-\varepsilon_{s}}{\varepsilon_{m}+2 \varepsilon_{s}}\right|^{2} \\
& C_{a b s}=k \operatorname{Im}[\alpha]=4 \pi k R^{3} \operatorname{Im}\left[\frac{\varepsilon_{m}-\varepsilon_{s}}{\varepsilon_{m}+2 \varepsilon_{s}}\right] \\
& C_{e x t}=\frac{9 \omega}{c} V \varepsilon_{s}^{3 / 2} \frac{\varepsilon_{2}}{\left[\varepsilon_{1}+2 \varepsilon_{s}\right]^{2}+\varepsilon_{2}^{2}}
\end{aligned}
$$

Equation (B.25) gives the extinction cross-section of a spherical metal nanoparticle. If the imaginary part of the dielectric function is very small, it can loosely be said that the resonance occurs when $\varepsilon_{1}$ becomes equal to $-2 \varepsilon_{s}$. The frequency of incident light incident on a metal nanosphere in air must be roughly equal to $1 / \sqrt{3}$ times the bulk plasma frequency, i.e., $\omega \approx \omega_{p b} / \sqrt{3}$ for resonance.

\section{CONFLICT OF INTEREST}

The authors confirm that this article content has no conflicts of interest.

\section{ACKNOWLEDGEMENTS}

The present work is partially supported by the Council of Scientific and Industrial Research (India).

\section{REFERENCES}

[1] Bardhan R, Lal S, Joshi A, Halas NJ. Theranostic nanoshells: From probe design to imaging and treatment of cancer. ACC Chem Res 2011; 44(10): 936-46

[2] Choi H, Pile DF, Nam S, Bartal G, Zhang X. Compressing surface plasmons for nanoscale optical focusing. Opt Express 2009; 17: 7519-24.

[3] Celebrano M, Lettow R, Kukura P, et al. Efficient coupling of single photons to single plasmons. Opt Exp 2010; 18: 13829-35.

[4] Stockman MI, Bergman DJ. Surface plasmon amplification by stimulated emission of radiation (SPASER). USA Patent No. 7,569,188, 2004.

[5] Zhu J, Ozdemir ŞK, Yang L. Sensors: Bypassing the diffusion limit. Nat Photon 2011; 5: 653-4.

[6] Kvasnička P, Chadt K, Vala M, Bocková M, Homola J. Toward single-molecule detection with sensors based on propagating surface plasmons. Opt Lett 2012; 37: 163-5.

[7] Gramotnev DK, Bozhevolny S. Plasmonics beyond the diffraction limit. Nat Photon 2010; 4: 83-91.

[8] Wood RW. On a remarkable case of uneven distribution of light in a diffraction grating spectrum. Philos Mag 1902; 4: 396-402.

[9] Zenneck J. Uber die fortpflanztmg ebener elektro-magnetischer wellen langs einer ebenen leiterflache und ihre beziehung zur drahtlosen telegraphie. Ann Phys 1907; 23: 846-66.

[10] Sommerfeld A. Propagation of waves in wireless telegraphy. Ann Phys 1909; 28: 665-736.

[11] Fano RM. The theory of anomalous diffraction grating of quasi stationary wave on metallic surfaces (Sommerfeld's waves). J Opt Soc Am 1941; 31: 213-22.

[12] Ritchie RH. Plasma losses by fast electrons in thin films. Phys Rev 1957; 106: 874-81.

[13] Turbadar T. Complete absorption of light by thin metal films. Proc Phys Soc 1959; 73: 40-4.

[14] Powell CJ, Swan JB. Effect of oxidation on the characteristic loss spectra of aluminum and magnesium. Phys Rev 1960; 118: 640-3.

[15] Stern EA, Ferrell RA. Surface plasma oscillation of degenerate electron gas. Phys Rev 1960; 120: 130-6.

[16] Otto A. Excitation of nonradiative surface plasma waves in silver by the method of frustrated total reflection. Zeit Phys 1968; 216: 398-410.

[17] Kretschmann E. Die bestimmung optischer konstanten von metallen durch anregung von vberflachenplasmashwingungen. Zeit Phys 1971; 241: 313-24. 
[18] Raether H. Surface plasmons on smooth and rough surfaces and on gratings, Berlin. Springer- Verlag 1988.

[19] Villuendas F, Pelayo J. Optical fibre device for chemical seeming based on surface plasmon excitridon. Sens Act A 1990; 23: 11425 .

[20] Aldea GC, Mateo J. Four-layer chemical fibre optic plasmon based sensor. Sens Act B 1992; 7: 771-4.

[21] Maria LD, Martinelli M, Vegetti G. Fiber-optic sensor based on surface plasmon interrogation. Sens Act B 1993; 12: 221-3.

[22] Jorgenson RC, Yee SS. A fiber-optic chemical sensor based on surface plasmon resonance. Sens Act B 1993; 12: 213-20.

[23] Jha R, Verma R, Gupta B. Surface plasmon resonance-based tapered fiber optic sensor: Sensitivity enhancement by introducing a teflon layer between core and metal layer. Plasmonics 2008; 3 : 151-6.

[24] Diez A, Andrés MV, Cruz JL. In-line fiber-optic sensors based on the excitation of surface plasma modes in metal-coated tapered fibers. Sens Act B 2001; 73: 95-9.

[25] Homola J. On the sensitivity of surface plasmon resonance sensors with spectral interrogation. Sens Act B 1997; 1: 207-11.

[26] Rajan Chand S, Gupta BD. Fabrication and characterization of a surface plasmon resonance based fiber-optic sensor for bittering component--Naringin. Sens Act B 2006; 115: 344-8.

[27] Slavík R, Homola J, Ctyroký J, Brynda E. Novel spectral fiber optic sensor based on surface plasmon resonance. Sens Act B 2001; 74: 106-11.

[28] Slavík R, Homola J, Brynda E. A miniature fiber optic surface plasmon resonance sensor for fast detection of staphylococcal enterotoxin B. Biosens Bioelectron 2002; 17: 591-5.

[29] Sharma AK, Gupta BD. On the performance of different bimetallic combinations in surface plasmon resonance based fiber optic sensors. J Appl Phys 2007; 101: 093111.

[30] Sharma AK, Gupta BD. Fibre-optic sensor based on surface plasmon resonance with Ag-Au alloy nanoparticle films. Nanotechnology 2006; $17: 124$

[31] Tripathi SM, Kumar A, Marin E, Meunier JP. Side-polished optical fiber grating-based refractive index sensors utilizing the pure surface plasmon polariton. Lightwave Technol 2008; 26: 1980-5.

[32] Ran Z, Rao Y, Zhang J, Liu Z, Xu B. A miniature fiber-optic refractive-index sensor based on laser-machined Fabry-Perot interferometer tip. J Lightwave Technol 2009; 27: 5426-9.

[33] Srivastava SK, Verma R, Gupta BD. Surface plasmon resonance based fiber optic sensor for the detection of low water content in ethanol. Sens Act B 2011; 153: 194-8.

[34] Riobóo RJ, Philipp M, Ramos M, Krüger J. Concentration and temperature dependence of the refractive index of ethanol-water mixtures: Influence of intermolecular interactions. Eur Phys J E L Soft Mater 2009; 30: 19-26.

[35] Mendes LS, Oliveira FCC, Suarez PAZ, Rubim JC. Determination of ethanol in fuel ethanol and beverages by Fourier transform (FT)near infrared and FT-Raman spectrometries. Anal Chim Act 2003; 493: 219-31

[36] Srivastava SK, Verma R, GuptaBD. Surface plasmon resonance based fiber optic glucose biosensor. Proc SPIE 2012; 8351: $83511 \mathrm{Z}$.

[37] Verma R, Srivastava SK, Gupta BD. Surface plasmon resonance based fiber optic sensor for the detection of low density lipoprotein. IEEE Sens J 2012;12 (12): 3460-6.

[38] Gao Y, Kyratzis I. Covalent immobilization of protein on carbon nanotubes using the cross-linker 1-ethyl-3-(3dimethylaminopropyl) carbodiimide- a critical assessment. Bioconjing Chem 2008; 19: 1945-50

[39] Lioubimov V, Kolomenskii A, Mershin A, Nanopoulos DV, Schuessler HA. Effect of varying electric potential on surfaceplasmon resonance sensing. Appl Opt 2004; 43: 3426-32.

[40] Sharma AK, Gupta BD. Influence of temperature on the sensitivity and signal-to-noise ratio of a fiber-optic surface-plasmon resonance sensor. Appl Opt 2006; 45: 151-61.

[41] Sharma AK, Rajan, Gupta BD. Influence of dopants on the performance of a fiber optic surface plasmon resonance sensor. Opt Commun 2007; 274: 320-6.

[42] Dwivedi YS, Sharma AK, Gupta BD. Influence of skew rays on the sensitivity and signal-to-noise ratio of a fiber-optic surfaceplasmon-resonance sensor: a theoretical study. Appl Opt 2007; 46: 4563-9.
[43] Srivastava SK, Gupta BD. Influence of ions on the surface plasmon resonance spectrum of a fiber optic refractive index sensor. Sens Act B 2011; 156: 559-62.

[44] Bueno FJ, Esteban O, Díaz-Herrera N, Navarrete MC, GonzálezCano A. Sensing properties of asymmetric double-layer-covered tapered fibers. Appl Opt 2004; 43: 1615-20.

[45] Kim YC, Peng W, Banerji S, Booksh KS. Tapered fiber optic surface plasmon resonance sensor for analyses of vapor and liquid phases. Opt Lett 2005; 30: 2218-20.

[46] Villatoro J, Monzón-Hernández D, Mejía E. Fabrication and modeling of uniform-waist single-mode tapered optical fiber sensors. Appl Opt 2003; 42: 2278-83

[47] Verma RK, Sharma AK, Gupta BD. Modeling of tapered fiberoptic surface plasmon resonance sensor with enhanced sensitivity. IEEE Photon Technol Lett 2007; 19: 1786-8.

[48] Verma RK, Sharma AK, Gupta BD. Surface plasmon resonance based tapered fiber optic sensor with different taper profiles. Opt Commun 2008; 281: 1486-91

[49] Verma RK, Gupta BD. Theoretical modelling of a bi-dimensional U-shaped surface plasmon resonance based fibre optic sensor for sensitivity enhancement. J Phys D Appl Phys 2008; 41: 095106.

[50] Srivastava SK, Gupta BD. A multitapered fiber-optic SPR sensor with enhanced sensitivity. IEEE Photon Technol Lett 2011; 23: 923-5.

[51] Bhatia P, Gupta BD. Surface-plasmon-resonance-based fiber-optic refractive index sensor: sensitivity enhancement. Appl Opt 2011; 50: 2032-6.

[52] Bhatia P, Gupta BD. Fabrication and characterization of a surface plasmon resonance based fiber optic urea sensor for biomedical applications. Sens Act B 2012; 161: 434-8.

[53] Daniel MC, Astruc D. Gold nanoparticles: assembly, supramolecular chemistry, quantum-size-related properties, and applications toward biology, catalysis, and nanotechnology. Chem Rev 2003; 104: 293-346.

[54] Wagner FE, Haslbeck S, Stievano L, Calogero S, Pankhurst QA, Martinek KP. Before striking gold in gold-ruby glass. Nature 2000; 407: 691-2.

[55] Piccolpasso C. Li tre libri dell'arte del vasajoa. Carolina: Nabu Press 2011

[56] Antonii F. Panacea aurea-auro potabile. Hamburg, Germany Bibliopolio Frobeniano, 1618

[57] Kunckels J. Nuetaliche observationes oder anmerkungen von auro und argento potabili. G. Schultzens, Hamburg: 1676.

[58] Cassius A. De auro. Hamburg Germany:1685.

[59] Helcher HH. Aurum potabile oder gold tincture. Breslau and Leipdg; J Herbord Klossen 1718.

[60] Fulhame Mrs. An essay on combustion with a view to a new art of dying and painting. London: J Cooper, 1794.

[61] Ostwald W. Zur geschichte des colloiden goldes. Kolloid Zeitschrift 1909; 4: 5-14.

[62] Faraday M. Experimental relations of gold (and other metals) to light. Philos Trans 1857; 147: 145-81.

[63] Turkevich J, Stevenson PC, Hillier J. A study of the nucleation and growth processes in the synthesis of colloidal gold. Discuss Faraday Soc 1951; 11: 55-75.

[64] Mie G. Beiträge zur optik trüber medien, speziell kolloidaler metallösungen. Ann Phys 1908; 330: 377-445.

[65] Kreibig U, Genzel L. Optical absorption of small metallic particles. Surf Sci 1985; 156: 678-700.

[66] Link S, El-Sayed MA. Size and temperature dependence of the plasmon absorption of colloidal gold nanoparticles. J Phys Chem B 1999; 103: 4212-7.

[67] Chau LK, Lin YF, Cheng SF, Lin TJ. Fiber-optic chemical and biochemical probes based on localized surface plasmon resonance. Sens Act B 2006; 113: 100-5.

[68] Lin TJ, Lou C T. Reflection-based localized surface plasmon resonance fiber-optic probe for chemical and biochemical sensing at high-pressure conditions. J Supercrit Fluids 2007; 41: 317-25.

[69] Lin TJ, Chung MF. Detection of cadmium by a fiber-optic biosensor based on localized surface plasmon resonance. Biosens Bioelectron 2009; 24: 1213-8.

[70] Sai VVR, Kundu T, Mukherji S. Novel U-bent fiber optic probe for localized surface plasmon resonance based biosensor. Biosens Bioelectron 2009; 24: 2804-9. 
[71] Srivastava SK, Verma RK, Gupta BD. Theoretical modeling of a localized surface plasmon resonance based intensity modulated fiber optic refractive index sensor. Appl Opt 2009; 48: 3796-802.

[72] Dhawan A, Muth JF. Plasmon resonances of gold nanoparticles incorporated inside an optical fibre matrix. Nanotechnology. 2006; 17: 2504-11.

[73] Ozdemir ŞK, Turhan-Sayan G. Temperature Effects on Surface Plasmon Resonance: Design Considerations for an Optical Temperature Sensor. J Lightw Technol 2003; 21: 805-14.

[74] Sharma AK, Gupta BD. Theoretical model of a fiber optic remote sensor based on surface plasmon resonance for temperature detection. Opt Fiber Technol 2006; 12: 87-100.

[75] Srivastava SK, Gupta BD. Simulation of a localized surfaceplasmon-resonance-based fiber optic temperature sensor. J Opt Soc Am A 2010; 27: 1743-9.

[76] Prabhakar S, Srivastava SK, Gupta BD, Mehta BR. A localized surface plasmon resonance based fiber optic hydrogen gas sensor. OSI Symposium on Fiber Optics and Photonics (FOP11), IIT Delhi, New Delhi, India 2011.

[77] Moreira CS, Lima AMN, Neff H, Thirstrup C. Temperaturedependent sensitivity of surface plasmon resonance sensors at the gold-water interface. Sens Act B 2008; 134: 854-62.

[78] Srivastava SK, Arora V, Sapra S, Gupta BD. Surface plasmon resonance based fiber optic sensor utilizing metal nanoparticles: Influence of ambient temperature. Sens Trans 2012; 139: 95-108.

[79] Khijwania SK, Gupta BD. Maximum achievable sensitivity of the fiber optic evanescent field absorption sensor based on the Ushaped probe. Opt Commun 2000; 175: 135-137.
[80] Srivastava SK, Arora V, Sapra S, Gupta BD. Localized surface plasmon resonance based fiber optic U-shaped biosensor for the detection of blood glucose. Plasmonics 2012; 7: 261-8.

[81] Verma R, Srivastava SK, Gupta BD. Surface plasmon resonance based multi-channel and multi-analyte fiber optic sensor. Proc. SPIE 2012; 8351: 83512D

[82] Kabashin AV, Evans P, Pastkovsky S, et al. Plasmonic nanorod metamaterials for biosensing. Nat Mater 2009; 8: 867-71.

[83] Huang C, Ye J, Wang S, Stakenborg T, Lagae L. Gold nanoring as a sensitive plasmonic biosensor for on-chip DNA detection. Appl Phys Lett 2012; 100: 173114.

[84] Prabhakar A, Mukherji S. A novel C-shaped, gold nanoparticle coated, embedded polymer waveguide for localized surface plasmon resonance based detection. Lab Chip 2010; 10: 3422-5.

[85] Shopova SI, Rajmangal R, Holler S, Arnold S. Plasmonic enhancement of a whispering-gallery-mode biosensor for single nanoparticle detection. Appl Phys Lett 2011; 98: 243103-4.

[86] Swaim JD, Knittel J, Bowen WP. Detection limits in whispering gallery biosensors with plasmonic enhancement. Appl Phys Lett 2011; 99: 243109-3.

[87] Raether H. Surface Plasmons on Smooth and Rough Surfaces and on Gratings. Berlin: Springer- Verlag, 1988

[88] Maier SA. Plasmonics: fundamentals and applications. USA: Springer 2007

[89] Bohren CF, Huffman DR. Absorption and scattering of light by small nanoparticles. Germany: Wiley - VCH, 1998.

[90] Jackson JD. Classical Electrodynamics. $3^{\text {rd }}$ ed. NY: John Wiley and Sons, Inc., 1999.

(C) Srivastava and Gupta; Licensee Bentham Open.

This is an open access article licensed under the terms of the Creative Commons Attribution Non-Commercial License (http://creativecommons.org/licenses/by-nc/3.0/) which permits unrestricted, non-commercial use, distribution and reproduction in any medium, provided the work is properly cited. 